\title{
LIPSCHITZ MATCHBOX MANIFOLDS
}

\author{
STEVEN HURDER
}

\begin{abstract}
A matchbox manifold is a connected, compact foliated space with totally disconnected transversals; or in other notation, a generalized lamination. It is said to be Lipschitz if there exists a metric on its transversals for which the holonomy maps are Lipschitz. Examples of Lipschitz matchbox manifolds include the exceptional minimal sets for $C^{1}$-foliations of compact manifolds, tiling spaces, the classical solenoids, and the weak solenoids of McCord and Schori, among others. We address the question: When does a Lipschitz matchbox manifold admit an embedding as a minimal set for a smooth dynamical system, or more generally for as an exceptional minimal set for a $C^{1}$-foliation of a smooth manifold? We gives examples which do embed, and develop criteria for showing when they do not embed, and give examples. We also discuss the classification theory for Lipschitz weak solenoids.
\end{abstract}

\section{Introduction}

In this paper, we consider the classification and embedding problems for matchbox manifolds, from the viewpoint of Lipschitz pseudogroups, and develop invariants which are obstructions to realizing a matchbox manifold as a minimal set. Matchbox manifolds are a class of continua that occur naturally in the study of dynamical systems, and in foliation theory as exceptional minimal sets. The overall goal of this research program is to develop tools for the classification of these spaces, and to understand which matchbox manifolds are homeomorphic to exceptional minimal sets for $C^{r}$-foliations, for $r \geq 1$.

We first discuss an important motivation for interest in this program of study.

PROBLEM 1.1 (Sondow [137). When is a smooth connected n-manifold L without boundary, diffeomorphic to a leaf of a foliation $\mathcal{F}_{M}$ of a compact smooth manifold $M$ ?

For the case where $L$ has dimension $n=1$, the problem is trivial. Also, for dimension $n=2$, Cantwell and Conlon showed in 29] that any surface without boundary is a diffeomorphic to a leaf of a smooth codimension-1 foliation of a compact 3-manifold.

On the other hand, Ghys [58] and Inaba, Nishimori, Takamura and Tsuchiya [82, constructed 3manifolds which are not homeomorphic to a leaf of any codimension- 1 foliation of a compact manifold. Souza and Schweitzer 135 give further examples in higher dimensions, of manifolds which cannot be leaves in codimension one. The non-embedding examples by these authors are essentially the only known results on Problem 1.1] in this generality, and they are for codimension-one foliations.

There is a natural variant of Problem 1.1, posed in the 1974 ICM address by Sullivan [138]:

PROBLEM 1.2. Let $L$ be a complete Riemannian smooth manifold without boundary. When is $L$ quasi-isometric to a leaf of a $C^{r}$-foliation $\mathcal{F}_{M}$ of a compact smooth manifold $M$, for $r \geq 1$ ?

A quasi-isometric embedding of $L$ must preserve its quasi-invariant geometric properties, which can be used to construct obstructions to such an embedding. For example, Cantwell and Conlon studied in [27, 28] how the asymptotic behavior of the metric on $L$ is related to the dynamics of the leaf in a codimension-one foliation.

2010 Mathematics Subject Classification. Primary 52C23, 57R05, 54F15, 37B45; Secondary 53C12, 57N55 .

Version date: September 5, 2013; updated August 26, 2014. 
The work of Phillips and Sullivan in [119] introduced the asymptotic Euler class of a non-compact Riemannian 2-manifold $L$ which has subexponential volume growth rate, and showed this can be used as an obstruction to a quasi-isometric embedding of $L$ as a leaf, depending on the topology of the ambient manifold $M$. This result was generalized by Januszkiewicz in 83 to obtain obstructions in terms of the asymptotic Pontrjagin numbers of an open Riemannian $n$-manifold with subexponential volume growth rate, for $n=4 k$ with $k \geq 1$.

In an alternate direction, Attie and Hurder in $[8$ introduced an invariant of open manifolds, its "leaf entropy", or "asymptotic leaf complexity", and gave examples of open manifolds with exponential volume growth rate that cannot be quasi-isometric to a leaf in a foliation of any codimension. Examples of surfaces with exponential growth rate that cannot be quasi-isometric to a leaf were constructed by Schweitzer in [133] and Zeghib in [152, using a variant of the approach in [8]. The work of Schweitzer [134] exhibits further examples of complete Riemannian manifolds which are not quasi-isometric to a leaf in any codimension-one foliation. The work of the author and Lukina [80] generalizes the results of [8] to the broader class of matchbox manifolds.

The non-embedding results mentioned above rely on the simple strategy, that a leaf in a compact foliated manifold $M$ has some type of recurrence properties, and the idea is to formulate such a property, intrinsic to $L$, which cannot be satisfied if $L$ is homeomorphic to a leaf, or possibly quasiisometric to a leaf. Each such criteria for non-recurrence then yields non-embeddability results.

A leaf $L$ contained in a minimal set $\mathfrak{M}$ for a foliation $\mathcal{F}_{M}$ on a compact manifold $M$ has much stronger recurrence properties. For example, Cass observed in [33] that such a leaf must be "quasihomogeneous", and that this property is an invariant of the quasi-isometry class of a Riemannian metric on $L$. He consequently gave examples of complete Riemannian manifolds, including leaves of foliations, which cannot be quasi-isometric to a leaf in a minimal set. For example, Cass showed that any non-compact leaf in a Reeb foliation of $\mathbb{S}^{3}$ cannot be realized as a leaf of a minimal set in any codimension.

The question raised by Cass' work suggests a variant of the above questions, where we consider the closure $\mathfrak{M}=\bar{L}$ of a non-compact leaf $L \subset M$, where $\mathfrak{M}$ has the structure of a foliated space. The formal definition of a foliated space $\mathfrak{M}$ was given by Moore and Schochet [114, Chapter 2], as part of their development of a general formulation of the Connes measured leafwise-index theorem [43]. Candel and Conlon [26. Chapter 11] further developed the theory of foliated spaces, and gave many interesting examples. We are particularly interested in those cases where the transverse model space for the foliated space $\mathfrak{M}$ is totally disconnected.

A compact connected foliated space $\mathfrak{M}$ with totally disconnected transversals is called a "matchbox manifold", in accordance with terminology introduced in continua theory [2, 3, 4]. A matchbox manifold with 2-dimensional leaves is a lamination by surfaces, as defined in [60, 101. If all leaves of $\mathfrak{M}$ are dense, then it is called a minimal matchbox manifold. A compact minimal set $\mathfrak{M} \subset M$ for a foliation $\mathcal{F}_{M}$ on a manifold $M$ yields a foliated space with foliation $\mathcal{F}=\mathcal{F} \mid \mathfrak{M}$. If the minimal set is exceptional, then $\mathfrak{M}$ is a minimal matchbox manifold. The formal definition and some basic properties of matchbox manifolds are discussed in Section 2 .

The leaves of the foliation $\mathcal{F}$ of a foliated space $\mathfrak{M}$ admit a smooth Riemannian metric, and for each leaf $L \subset \mathfrak{M}$ there is a well-defined quasi-isometry class of Riemannian metrics on $L$. The obstructions used in the works above, to show that a particular Riemannian manifold $L$ cannot be quasi-isometric to a leaf of a foliation of a compact manifold $M$, also provide obstructions to realizing $L$ as a leaf in a compact foliated space $\mathfrak{M}$.

The following problem is addressed in this work:

PROBLEM 1.3. Let $\mathfrak{M}$ be a minimal matchbox manifold. Does there exists a homeomorphism of $\mathfrak{M}$ to an exceptional minimal set of a $C^{r}$-foliation $\mathcal{F}_{M}$ of a manifold $M$, for $r \geq 1$ ?

When such an embedding exists, then each leaf $L \subset \mathfrak{M}$ is quasi-isometric to a leaf of $\mathcal{F}_{M}$. If the leaf $L$ is dense in $\mathfrak{M}$ and $\mathfrak{M}$ is non-embeddable, then this gives a criteria for the non-embedding 
of $L$, that depends not just on the intrinsic geometry and topology of $L$, but includes "extrinsic properties" of $L$ in $\mathfrak{M}$, such as the transverse geometry and dynamics of the foliated space $\mathfrak{M}$.

Observe that if $\mathfrak{M}$ is an invariant set for a $C^{r}$-foliation $\mathcal{F}_{M}$ of a Riemannian manifold $M$, where $r \geq 1$, then the holonomy maps for the foliation $\mathcal{F}$ on $\mathfrak{M}$ are induced by the holonomy maps of $\mathcal{F}_{M}$, and there is a metric on the transversals to $\mathfrak{M}$ such that the holonomy maps of $\mathcal{F}$ are Lipschitz, as discussed in Section 4. Problem 1.3 can be thus be reformulated as.

PROBLEM 1.4. Let $\mathfrak{M}$ be a Lipschitz matchbox manifold. Find obstructions to the existence of a foliated Lipschitz embedding $\iota: \mathfrak{M} \rightarrow M$, where $M$ has a $C^{r}$-foliation $\mathcal{F}_{M}$ with $r \geq 1$.

This problem can also be considered as asking for a characterization of the Lipschitz structures which can arise for the transverse Cantor sets to exotic minimal sets in $C^{r}$-foliations. For example, in the case of a foliation obtained from the suspension of a diffeomorphism of the circle $\mathbb{S}^{1}$, McDuff studied in [106] the question: which Cantor sets embedded in $\mathbb{S}^{1}$ are the invariant sets for $C^{1+\alpha_{-}}$ diffeomorphisms of the circle?

The general observations and results of this paper are combined in Section 8 to yield the following non-embedding results.

THEOREM 1.5. There exist Lipshitz matchbox manifolds which are not homeomorphic to the minimal set of any $C^{1}$-foliation.

THEOREM 1.6. There exist minimal matchbox manifolds which are not homeomorphic to the minimal set of any $C^{1}$-foliation.

Many further questions and problems are posed throughout the text, which is organized as follows.

Section 2 collects together some definitions and results concerning matchbox manifolds that we use in the paper. More details can be found in the works 26, 38, 39, 40, 114. Section 2 is rather dense, and can be skipped if the reader is only interested in Cantor pseudogroup actions.

Section 3 gives some some definitions concerning the dynamical properties of Cantor pseudogroup actions. Then in Section 4 the Lipschitz property for pseudogroup actions is introduced. The main result of this section is a proof that an embedding of a matchbox manifold as an exceptional minimal set in a $C^{1}$-foliation yields a Lipschitz structure on it. Section 5 discusses some examples from the literature of embeddings of matchbox manifolds as exceptional minimal sets for foliations.

In Section 6, the notion of normal, weak and generalized solenoids are introduced. These are basic examples for the study of minimal matchbox manifolds.

Section 7 introduces an operation on minimal matchbox manifolds, called their "fusion", which amalgamates their pseudogroups. The fusion process is inspired by the method introduced by Lukina in 99. The fusion process is used to construct the examples in Section 8 of minimal pseudogroup Cantor actions, which cannot be homeomorphic to an exceptional minimal set in any $C^{1}$-foliation.

Finally, in Section 9. Morita equivalence and Lipschitz equivalence of minimal Lipschitz pseudogroups are introduced. The problem of the classification of matchbox manifolds up to Lipschitz equivalence is considered for the the special case of weak solenoids.

\section{Foliated SPACES AND MATChBox MANifoldS}

We recall the notions of foliated spaces and matchbox manifolds, and their basic properties. The book by Moore and Schochet in [114. Chapter 2] introduced foliated spaces, as part of their development of a general form of the Connes measured leafwise index theorem. The textbook by Candel and Conlon [26. Chapter 11] further develops the theory, with many examples. Matchbox manifolds are a special class of connected foliated spaces, which have totally disconnected transversal spaces. The papers [37, 38, 39, 40, 41, 42 discuss the topology and dynamics of matchbox manifolds, especially with the goal of classifying these spaces up to homeomorphism. 
First we recall some basic notions. A topological space $\Omega$ is a continuum, if it is compact, connected, and metrizable. A Cantor set $\mathfrak{X}$ is a non-empty, compact, perfect and totally disconnected set. A set $V \subset \mathfrak{X}$ is clopen if it is both open and closed, and a topological space is totally disconnected if and only if it admits a basis for its topology consisting of clopen sets.

The definition of a foliated space is modeled on the definition of a smooth foliation.

DEFINITION 2.1. A foliated space of dimension $n$ is a compact metric space $\mathfrak{M}$, such that there exists a separable metric space $\mathfrak{X}$, and for each $x \in \mathfrak{M}$ there is a compact subset $\mathfrak{X}_{x} \subset \mathfrak{X}$, an open subset $U_{x} \subset \mathfrak{M}$, and a homeomorphism defined on the closure $\varphi_{x}: \bar{U}_{x} \rightarrow[-1,1]^{n} \times \mathfrak{X}_{x}$ such that $\varphi_{x}(x)=\left(0, w_{x}\right)$ where $w_{x} \in \operatorname{int}\left(\mathfrak{X}_{x}\right)$. Moreover, it is assumed that each $\varphi_{x}$ admits an extension to a foliated homeomorphism $\widehat{\varphi}_{x}: \widehat{U}_{x} \rightarrow(-2,2)^{n} \times \mathfrak{X}_{x}$ where $\bar{U}_{x} \subset \widehat{U}_{x}$. The space $\mathfrak{X}_{x}$ is called the local transverse model at $x$.

Let $\pi_{x}: \bar{U}_{x} \rightarrow \mathfrak{X}_{x}$ denote the composition of $\varphi_{x}$ with projection onto the second factor. For $w \in \mathfrak{X}_{x}$ the set $\mathcal{P}_{x}(w)=\pi_{x}^{-1}(w) \subset \bar{U}_{x}$ is called a plaque for the coordinate chart $\varphi_{x}$. We adopt the notation, for $z \in \bar{U}_{x}$, that $\mathcal{P}_{x}(z)=\mathcal{P}_{x}\left(\pi_{x}(z)\right)$, so that $z \in \mathcal{P}_{x}(z)$. Note that each plaque $\mathcal{P}_{x}(w)$ for $w \in \mathfrak{X}_{x}$ is given the topology so that the restriction $\varphi_{x}: \mathcal{P}_{x}(w) \rightarrow[-1,1]^{n} \times\{w\}$ is a homeomorphism. Then $\operatorname{int}\left(\mathcal{P}_{x}(w)\right)=\varphi_{x}^{-1}\left((-1,1)^{n} \times\{w\}\right)$. Let $U_{x}=\operatorname{int}\left(\bar{U}_{x}\right)=\varphi_{x}^{-1}\left((-1,1)^{n} \times \operatorname{int}\left(\mathfrak{X}_{x}\right)\right)$. Note that if $z \in U_{x} \cap U_{y}$, then $\operatorname{int}\left(\mathcal{P}_{x}(z)\right) \cap \operatorname{int}\left(\mathcal{P}_{y}(z)\right)$ is an open subset of both $\mathcal{P}_{x}(z)$ and $\mathcal{P}_{y}(z)$. The collection of sets

$$
\mathcal{V}=\left\{\varphi_{x}^{-1}(V \times\{w\}) \mid x \in \mathfrak{M}, w \in \mathfrak{X}_{x}, V \subset(-1,1)^{n} \text { open }\right\}
$$

forms the basis for the fine topology of $\mathfrak{M}$. The connected components of the fine topology are called leaves, and define the foliation $\mathcal{F}$ of $\mathfrak{M}$. Let $L_{x} \subset \mathfrak{M}$ denote the leaf of $\mathcal{F}$ containing $x \in \mathfrak{M}$.

DEFINITION 2.2. A smooth foliated space is a foliated space $\mathfrak{M}$ as above, for which there exists a choice of local charts $\varphi_{x}: \bar{U}_{x} \rightarrow[-1,1]^{n} \times \mathfrak{X}_{x}$ such that for all $x, y \in \mathfrak{M}$ with $z \in U_{x} \cap U_{y}$, there exists an open set $z \in V_{z} \subset U_{x} \cap U_{y}$ such that $\mathcal{P}_{x}(z) \cap V_{z}$ and $\mathcal{P}_{y}(z) \cap V_{z}$ are connected open sets, and the composition $\psi_{x, y ; z} \equiv \varphi_{y} \circ \varphi_{x}^{-1}: \varphi_{x}\left(\mathcal{P}_{x}(z) \cap V_{z}\right) \rightarrow \varphi_{y}\left(\mathcal{P}_{y}(z) \cap V_{z}\right)$ is a smooth map, where $\varphi_{x}\left(\mathcal{P}_{x}(z) \cap V_{z}\right) \subset \mathbb{R}^{n} \times\{w\} \cong \mathbb{R}^{n}$ and $\varphi_{y}\left(\mathcal{P}_{y}(z) \cap V_{z}\right) \subset \mathbb{R}^{n} \times\left\{w^{\prime}\right\} \cong \mathbb{R}^{n}$. The maps $\psi_{x, y ; z}$ are assumed to depend continuously on $z$ in the $C^{\infty}$-topology on maps between subsets of $\mathbb{R}^{n}$.

A map $f: \mathfrak{M} \rightarrow \mathbb{R}$ is said to be smooth if for each flow box $\varphi_{x}: \bar{U}_{x} \rightarrow[-1,1]^{n} \times \mathfrak{X}_{x}$ and $w \in \mathfrak{X}_{x}$ the composition $y \mapsto f \circ \varphi_{x}^{-1}(y, w)$ is a smooth function of $y \in(-1,1)^{n}$, and depends continuously on $w$ in the $C^{\infty}$-topology on maps of the plaque coordinates $y$. As noted in [114] and [26, Chapter 11], this allows one to define smooth partitions of unity, vector bundles, and tensors for smooth foliated spaces. In particular, one can define leafwise Riemannian metrics. We recall a standard result, whose proof for foliated spaces can be found in [26, Theorem 11.4.3].

THEOREM 2.3. Let $\mathfrak{M}$ be a smooth foliated space. Then there exists a leafwise Riemannian metric for $\mathcal{F}$, such that for each $x \in \mathfrak{M}, L_{x}$ inherits the structure of a complete Riemannian manifold with bounded geometry, and the Riemannian geometry of $L_{x}$ depends continuously on $x$. In particular, each leaf $L_{x}$ has the structure of a complete Riemannian manifold with bounded geometry.

Bounded geometry implies, for example, that for each $x \in \mathfrak{M}$, there is a leafwise exponential map $\exp _{x}^{\mathcal{F}}: T_{x} \mathcal{F} \rightarrow L_{x}$ which is a surjection, and the composition $\exp _{x}^{\mathcal{F}}: T_{x} \mathcal{F} \rightarrow L_{x} \subset \mathfrak{M}$ depends continuously on $x$ in the compact-open topology on maps.

DEFINITION 2.4. A matchbox manifold is a smooth foliated connected space $\mathfrak{M}$, such that its transverse model space $\mathfrak{X}$ is totally disconnected, and for each $x \in \mathfrak{M}$, the transverse model space $\mathfrak{X}_{x} \subset \mathfrak{X}$ in Definition 2.1 is a clopen subset.

All matchbox manifolds are assumed to be smooth with a given leafwise Riemannian metric. The space $\mathfrak{M}$ is assumed to be metrizable, and we fix a choice for the metric $d_{\mathfrak{M}}$ on $\mathfrak{M}$. The leafwise Riemannian metric $d_{\mathcal{F}}$ is continuous with respect to the metric $d_{\mathfrak{M}}$ on $\mathfrak{M}$, but otherwise the two metrics can be chosen independently. The metric $d_{\mathfrak{M}}$ is used to define the metric topology on $\mathfrak{M}$, while the metric $d_{\mathcal{F}}$ depends on an independent choice of the Riemannian metric on leaves. 
An important difference between a foliated matchbox manifold and a smooth foliated manifold is that the local foliation charts for a matchbox manifold are not connected, and so must be chosen appropriately to ensure that each chart is "local". We introduce the following conventions.

For $x \in \mathfrak{M}$ and $\epsilon>0$, let $D_{\mathfrak{M}}(x, \epsilon)=\left\{y \in \mathfrak{M} \mid d_{\mathfrak{M}}(x, y) \leq \epsilon\right\}$ be the closed $\epsilon$-ball about $x$ in $\mathfrak{M}$, and $B_{\mathfrak{M}}(x, \epsilon)=\left\{y \in \mathfrak{M} \mid d_{\mathfrak{M}}(x, y)<\epsilon\right\}$ the open $\epsilon$-ball about $x$.

Similarly, for $w \in \mathfrak{X}$ and $\epsilon>0$, let $D_{\mathfrak{X}}(w, \epsilon)=\left\{w^{\prime} \in \mathfrak{X} \mid d_{\mathfrak{X}}\left(w, w^{\prime}\right) \leq \epsilon\right\}$ be the closed $\epsilon$-ball about $w$ in $\mathfrak{X}$, and $B_{\mathfrak{X}}(w, \epsilon)=\left\{w^{\prime} \in \mathfrak{X} \mid d_{\mathfrak{X}}\left(w, w^{\prime}\right)<\epsilon\right\}$ the open $\epsilon$-ball about $w$.

Given a leaf $L$ and a piecewise $C^{1}$-path $\gamma:[0,1] \rightarrow L$, let $\|\gamma\|_{\mathcal{F}}$ denote its path-length for the leafwise Riemannian metric. Then give $L \subset \mathfrak{M}$ the path-length metric: if $x, y \in L$ then set

$d_{\mathcal{F}}(x, y)=\inf \left\{\|\gamma\|_{\mathcal{F}} \mid \gamma:[0,1] \rightarrow L\right.$ is piecewise $\left.\mathrm{C}^{1}, \gamma(0)=x, \gamma(1)=y, \gamma(t) \in L \quad \forall 0 \leq t \leq 1\right\}$, and otherwise, if $x, y \in \mathfrak{M}$ are not on the same leaf, then set $d_{\mathcal{F}}(x, y)=\infty$.

For each $x \in \mathfrak{M}$ and $r>0$, let $D_{\mathcal{F}}(x, r)=\left\{y \in L_{x} \mid d_{\mathcal{F}}(x, y) \leq r\right\}$.

For each $x \in \mathfrak{M}$, the Gauss Lemma implies that there exists $\lambda_{x}>0$ such that $D_{\mathcal{F}}\left(x, \lambda_{x}\right)$ is a strongly convex subset for the metric $d_{\mathcal{F}}$. That is, for any pair of points $y, y^{\prime} \in D_{d_{\mathcal{F}}}\left(x, \lambda_{x}\right)$ there is a unique shortest geodesic segment in $L_{x}$ joining $y$ and $y^{\prime}$ and contained in $D_{\mathcal{F}}\left(x, \lambda_{x}\right)$ (cf. [50. Chapter 3, Proposition 4.2], or [67. Theorem 9.9]). Then for all $0<\lambda<\lambda_{x}$ the disk $D_{\mathcal{F}}(x, \lambda)$ is also strongly convex. The leafwise metrics have uniformly bounded geometry, so we obtain:

LEMMA 2.5. There exists $\lambda_{\mathcal{F}}>0$ such that for all $x \in \mathfrak{M}, D_{\mathcal{F}}\left(x, \lambda_{\mathcal{F}}\right)$ is strongly convex.

The following proposition summarizes results in [38, sections $2.1-2.2]$.

PROPOSITION 2.6. For a smooth foliated space $\mathfrak{M}$, given $\epsilon_{\mathfrak{M}}>0$, there exist constants $\lambda_{\mathcal{F}}>0$ and $0<\delta_{\mathcal{U}}^{\mathcal{F}}<\lambda_{\mathcal{F}} / 5$, and a covering of $\mathfrak{M}$ by foliation charts $\left\{\varphi_{i}: \bar{U}_{i} \rightarrow[-1,1]^{n} \times \mathfrak{X}_{i} \mid 1 \leq i \leq \nu\right\}$ with the following properties: For each $1 \leq i \leq \nu$, let $\pi_{i}=\pi_{x_{i}}: \bar{U}_{i} \rightarrow \mathfrak{X}_{i}$ be the projection, then

(1) Interior: $U_{i} \equiv \operatorname{int}\left(\bar{U}_{i}\right)=\varphi_{i}^{-1}\left((-1,1)^{n} \times B_{\mathfrak{X}}\left(w_{i}, \epsilon_{i}\right)\right)$, where $w_{i} \in \mathfrak{X}_{i}$ and $\epsilon_{i}>0$.

(2) Locality: for $x_{i} \equiv \varphi_{i}^{-1}\left(w_{i}, 0\right) \in \mathfrak{M}, \bar{U}_{i} \subset B_{\mathfrak{M}}\left(x_{i}, \epsilon_{\mathfrak{M}}\right)$.

For $z \in \bar{U}_{i}$, the plaque of the chart $\varphi_{i}$ through $z$ is denoted by $\mathcal{P}_{i}(z)=\mathcal{P}_{i}\left(\pi_{i}(z)\right) \subset \bar{U}_{i}$.

(3) Convexity: the plaques of $\varphi_{i}$ are strongly convex subsets for the leafwise metric.

(4) Uniformity: for $w \in \mathfrak{X}_{i}$ let $x_{w}=\varphi_{x_{i}}^{-1}(0, w)$, then

$$
D_{\mathcal{F}}\left(x_{w}, \delta_{\mathcal{U}}^{\mathcal{F}} / 2\right) \subset \mathcal{P}_{i}(w) \subset D_{\mathcal{F}}\left(x_{w}, \delta_{\mathcal{U}}^{\mathcal{F}}\right)
$$

(5) The projection $\pi_{i}\left(U_{i} \cap U_{j}\right)=\mathfrak{X}_{i, j} \subset \mathfrak{X}_{i}$ is a clopen subset for all $1 \leq i, j \leq \nu$.

A regular foliated covering of $\mathfrak{M}$ is one that satisfies the above conditions (2.6.1) to (2.6.5).

This technical result highlights one of the main issues with foliated spaces and matchbox manifolds, as contrasted with smooth foliations of compact manifolds, one has to assume or prove for these more exotic foliated spaces many of the regularity properties that are used in the study of foliations.

We assume in the following that a regular foliated covering of $\mathfrak{M}$ as in Proposition 2.6 has been chosen. Let $\mathcal{U}=\left\{U_{1}, \ldots, U_{\nu}\right\}$ denote the corresponding open covering of $\mathfrak{M}$. We can assume that the spaces $\mathfrak{X}_{i}$ form a disjoint clopen covering of $\mathfrak{X}$, so that $\mathfrak{X}=\mathfrak{X}_{1} \cup \dot{\cup} \cdots \dot{\cup} \mathfrak{X}_{\nu}$.

Let $\epsilon_{\mathcal{U}}>0$ be a Lebesgue number for $\mathcal{U}$. That is, given any $z \in \mathfrak{M}$ there exists some index $1 \leq i_{z} \leq \nu$ such that the open metric ball $B_{\mathfrak{M}}\left(z, \epsilon_{\mathcal{U}}\right) \subset U_{i_{z}}$.

For $1 \leq i \leq \nu$, let $\lambda_{i}: \bar{U}_{i} \rightarrow[-1,1]^{n}$ be the projection, so that for each $z \in U_{i}$ the restriction $\lambda_{i}: \mathcal{P}_{i}(z) \rightarrow[-1,1]^{n}$ is is a smooth coordinate system on the plaque.

For each $1 \leq i \leq \nu$ the set $\mathcal{T}_{i}=\varphi_{i}^{-1}\left(0, \mathfrak{X}_{i}\right)$ is a compact transversal to $\mathcal{F}$. Without loss of generality, we can assume that the transversals $\left\{\mathcal{T}_{1}, \ldots, \mathcal{T}_{\nu}\right\}$ are pairwise disjoint in $\mathfrak{M}$. Then define sections

$$
\tau_{i}: \mathfrak{X}_{i} \rightarrow \bar{U}_{i} \text {, defined by } \tau_{i}(\xi)=\varphi_{i}^{-1}(0, \xi) \text {, so that } \pi_{i}\left(\tau_{i}(\xi)\right)=\xi \text {. }
$$


Then $\mathcal{T}_{i}=\mathcal{T}_{x_{i}}$ is the image of $\tau_{i}$ and we let $\mathcal{T}=\mathcal{T}_{1} \cup \cdots \cup \mathcal{T}_{\nu} \subset \mathfrak{M}$ denote their disjoint union, and $\tau: \mathfrak{X} \rightarrow \mathcal{T}$ the union of the maps $\tau_{i}$.

A map $f: \mathfrak{M} \rightarrow \mathfrak{M}^{\prime}$ between foliated spaces is said to be a foliated map if the image of each leaf of $\mathcal{F}$ is contained in a leaf of $\mathcal{F}^{\prime}$. If $\mathfrak{M}^{\prime}$ is a matchbox manifold, then each leaf of $\mathcal{F}$ is path connected, so its image is path connected, hence must be contained in a leaf of $\mathcal{F}^{\prime}$. Thus,

LEMMA 2.7. Let $\mathfrak{M}$ and $\mathfrak{M}^{\prime}$ be matchbox manifolds, and $f: \mathfrak{M}^{\prime} \rightarrow \mathfrak{M}$ a continuous map. Then $f$ maps the leaves of $\mathcal{F}^{\prime}$ to leaves of $\mathcal{F}$. In particular, any homeomorphism $f: \mathfrak{M}^{\prime} \rightarrow \mathfrak{M}$ of matchbox manifolds is a foliated map.

A leafwise path is a continuous map $\gamma:[0,1] \rightarrow \mathfrak{M}$ such that there is a leaf $L$ of $\mathcal{F}$ for which $\gamma(t) \in L$ for all $0 \leq t \leq 1$. If $\mathfrak{M}$ is a matchbox manifold, and $\gamma:[0,1] \rightarrow \mathfrak{M}$ is continuous, then $\gamma$ is a leafwise path by Lemma 2.7. In the following, we will assume that all paths are piecewise differentiable.

The holonomy pseudogroup of a smooth foliated manifold $(M, \mathcal{F})$ generalizes the concept of a Poincaré section for a flow, which induces a discrete dynamical system associated to the flow. Associated to a leafwise path $\gamma$ is a holonomy map $h_{\gamma}$, which is a local homeomorphism on the transversal space. For a matchbox manifold $(\mathfrak{M}, \mathcal{F})$ the holonomy along a leafwise path is defined analogously. We briefly recall below the ideas and notations of the construction of holonomy maps for matchbox manifolds; further details and proofs are given in [38, 39].

A pair of indices $(i, j), 1 \leq i, j \leq \nu$, is said to be admissible if $U_{i} \cap U_{j} \neq \emptyset$. For $(i, j)$ admissible, set $\mathfrak{X}_{i, j}=\pi_{i}\left(U_{i} \cap U_{j}\right) \subset \mathfrak{X}_{i}$. The regularity of foliation charts imply that plaques are either disjoint, or have connected intersection. For $(i, j)$ admissible, there is a well-defined transverse change of coordinates homeomorphism $h_{i, j}: \mathfrak{X}_{i, j} \rightarrow \mathfrak{X}_{j, i}$ with domain $\operatorname{Dom}\left(h_{i, j}\right)=\mathfrak{X}_{i, j}$ and range $R\left(h_{i, j}\right)=\operatorname{Dom}\left(h_{j, i}\right)=\mathfrak{X}_{j, i}$. By definition they satisfy $h_{i, i}=I d, h_{i, j}^{-1}=h_{j, i}$, and if $U_{i} \cap U_{j} \cap U_{k} \neq \emptyset$ then $h_{k, j} \circ h_{j, i}=h_{k, i}$ on their common domain of definition. Note that the domain and range of $h_{i, j}$ are clopen subsets of $\mathfrak{X}$ by Proposition 2.615,

Recall that for $1 \leq i \leq \nu, \tau_{i}: \mathfrak{X}_{i} \rightarrow \mathcal{T}_{i}$ denotes the transverse section for the coordinate chart $U_{i}$, where $\mathcal{T}=\mathcal{T}_{1} \cup \cdots \cup \mathcal{T}_{\nu} \subset \mathfrak{M}$ denotes their disjoint union, and $\pi: \mathcal{T} \rightarrow \mathfrak{X}$ is the coordinate projection restricted to $\mathcal{T}$ which is a homeomorphism, with $\tau: \mathfrak{X} \rightarrow \mathcal{T}$ its inverse.

The holonomy pseudogroup $\mathcal{G}_{\mathcal{F}}$ of $\mathcal{F}$ is the topological pseudogroup modeled on $\mathfrak{X}$ generated by the elements of $\mathcal{G}_{\mathcal{F}}^{(1)}=\left\{h_{j, i} \mid(i, j)\right.$ admissible $\}$. We also define a subpseudogroup $\mathcal{G}_{\mathcal{F}}^{*} \subset \mathcal{G}_{\mathcal{F}}$ which is based on the holonomy along paths. A sequence $\mathcal{I}=\left(i_{0}, i_{1}, \ldots, i_{\alpha}\right)$ is admissible if each pair $\left(i_{\ell-1}, i_{\ell}\right)$ is admissible for $1 \leq \ell \leq \alpha$, and the composition $h_{\mathcal{I}}=h_{i_{\alpha}, i_{\alpha-1}} \circ \cdots \circ h_{i_{1}, i_{0}}$ has non-empty domain $\operatorname{Dom}\left(h_{\mathcal{I}}\right)$, which is defined to be the maximal clopen subset of $\mathfrak{X}_{i_{0}}$ for which the compositions are defined. Given a open subset $U \subset \operatorname{Dom}\left(h_{\mathcal{I}}\right)$ define the restriction $h_{\mathcal{I}} \mid U \in \mathcal{G}_{\mathcal{F}}$. Introduce

$$
\mathcal{G}_{\mathcal{F}}^{*}=\left\{h_{\mathcal{I}}|U| \mathcal{I} \text { admissible and } U \subset \operatorname{Dom}\left(h_{\mathcal{I}}\right)\right\} \subset \mathcal{G}_{\mathcal{F}} \text {. }
$$

The range of $g=h_{\mathcal{I}} \mid U$ is the open set $R(g)=h_{\mathcal{I}}(U) \subset \mathfrak{X}_{i_{\alpha}} \subset \mathfrak{X}$. Note that each map $g \in \mathcal{G}_{\mathcal{F}}^{*}$ admits a continuous extension $\bar{g}: \overline{\operatorname{Dom}(g)}=\bar{U} \rightarrow \mathfrak{X}_{i_{\alpha}}$ as $\operatorname{Dom}\left(h_{\mathcal{I}}\right)$ is a clopen set for each $\mathcal{I}$.

Let $\mathcal{I}=\left(i_{0}, i_{1}, \ldots, i_{\alpha}\right)$ be an admissible sequence. For each $1 \leq \ell \leq \alpha$, set $\mathcal{I}_{\ell}=\left(i_{0}, i_{1}, \ldots, i_{\ell}\right)$, and let $h_{\mathcal{I}_{\ell}}$ denote the corresponding holonomy map. For $\ell=0$, let $\mathcal{I}_{0}=\left(i_{0}, i_{0}\right)$. Note that $h_{\mathcal{I}_{\alpha}}=h_{\mathcal{I}}$ and $h_{\mathcal{I}_{0}}=I d: \mathfrak{X}_{0} \rightarrow \mathfrak{X}_{0}$.

Given $w \in \operatorname{Dom}\left(h_{\mathcal{I}}\right)$, let $x=\tau_{i_{0}}(w) \in L_{w}$. For each $0 \leq \ell \leq \alpha$, set $w_{\ell}=h_{\mathcal{I}_{\ell}}(w)$ and $x_{\ell}=\tau_{i_{\ell}}\left(w_{\ell}\right)$. Recall that $\mathcal{P}_{i_{\ell}}\left(x_{\ell}\right)=\mathcal{P}_{i_{\ell}}\left(w_{\ell}\right)$, where each $\mathcal{P}_{i_{\ell}}\left(w_{\ell}\right)$ is a strongly convex subset of the leaf $L_{w}$ in the leafwise metric $d_{\mathcal{F}}$. Introduce the plaque chain

$$
\mathcal{P}_{\mathcal{I}}(w)=\left\{\mathcal{P}_{i_{0}}\left(w_{0}\right), \mathcal{P}_{i_{1}}\left(w_{1}\right), \ldots, \mathcal{P}_{i_{\alpha}}\left(w_{\alpha}\right)\right\}
$$

Adopt the notation $\mathcal{P}_{\mathcal{I}}(x) \equiv \mathcal{P}_{\mathcal{I}}(w)$. Intuitively, a plaque chain $\mathcal{P}_{\mathcal{I}}(x)$ is a sequence of successively overlapping convex "tiles" in $L_{w}$ starting at $x=\tau_{i_{0}}(w)$, ending at $y=x_{\alpha}=\tau_{i_{\alpha}}\left(w_{\alpha}\right)$, and with each $\mathcal{P}_{i_{\ell}}\left(x_{\ell}\right)$ "centered" on the point $x_{\ell}=\tau_{i_{\ell}}\left(w_{\ell}\right)$.

Let $\gamma:[0,1] \rightarrow \mathfrak{M}$ be a path. Set $x_{0}=\gamma(0) \in U_{i_{0}}, w=\pi\left(x_{0}\right)$ and $x=\tau(w) \in \mathcal{T}_{i_{0}}$. Let $\mathcal{I}$ be an admissible sequence with $w \in \operatorname{Dom}\left(h_{\mathcal{I}}\right)$. We say that $(\mathcal{I}, w)$ covers $\gamma$ if the domain of $\gamma$ admits a 
partition $0=s_{0}<s_{1}<\cdots<s_{\alpha}=1$ such that $\mathcal{P}_{\mathcal{I}}(w)$ satisfies

$$
\gamma\left(\left[s_{\ell}, s_{\ell+1}\right]\right) \subset \mathcal{P}_{i_{\ell}}\left(\xi_{\ell}\right), 0 \leq \ell<\alpha \text {, and } \gamma(1) \in \mathcal{P}_{i_{\alpha}}\left(\xi_{\alpha}\right)
$$

For a path $\gamma$, we construct an admissible sequence $\mathcal{I}=\left(i_{0}, i_{1}, \ldots, i_{\alpha}\right)$ with $w \in \operatorname{Dom}\left(h_{\mathcal{I}}\right)$ so that $(\mathcal{I}, w)$ covers $\gamma$, and has "uniform domains". Inductively choose a partition of the interval $[0,1]$, say $0=s_{0}<s_{1}<\cdots<s_{\alpha}=1$, such that for each $0 \leq \ell \leq \alpha$,

$$
\gamma\left(\left[s_{\ell}, s_{\ell+1}\right]\right) \subset D_{\mathcal{F}}\left(x_{\ell}, \epsilon_{\mathcal{U}}^{\mathcal{F}}\right) \quad, \quad x_{\ell}=\gamma\left(s_{\ell}\right) .
$$

As a notational convenience, we have let $s_{\alpha+1}=s_{\alpha}$, so that $\gamma\left(\left[s_{\alpha}, s_{\alpha+1}\right]\right)=x_{\alpha}$. Choose $s_{\ell+1}$ to be the largest value of $s_{\ell}<s \leq 1$ such that $d_{\mathcal{F}}\left(\gamma\left(s_{\ell}\right), \gamma(t)\right) \leq \epsilon_{\mathcal{U}}^{\mathcal{F}}$ for all $s_{\ell} \leq t \leq s$, then $\alpha \leq\|\gamma\| / \epsilon_{\mathcal{U}}^{\mathcal{F}}$.

For each $0 \leq \ell \leq \alpha$, choose an index $1 \leq i_{\ell} \leq \nu$ so that $B_{\mathfrak{M}}\left(x_{\ell}, \epsilon_{\mathcal{U}}\right) \subset U_{i_{\ell}}$. Note that, for all $s_{\ell} \leq t \leq s_{\ell+1}, B_{\mathfrak{M}}\left(\gamma(t), \epsilon_{\mathcal{U}} / 2\right) \subset U_{i_{\ell}}$, so that $x_{\ell+1} \in U_{i_{\ell}} \cap U_{i_{\ell+1}}$. It follows that $\mathcal{I}_{\gamma}=\left(i_{0}, i_{1}, \ldots, i_{\alpha}\right)$ is an admissible sequence. Set $h_{\gamma}=h_{\mathcal{I}_{\gamma}}$ and note that $h_{\gamma}(w)=w^{\prime}$.

Next, consider paths $\gamma, \gamma^{\prime}:[0,1] \rightarrow \mathfrak{M}$ with $x=\gamma(0)=\gamma^{\prime}(0)$ and $y=\gamma(1)=\gamma^{\prime}(1)$. Suppose that $\gamma$ and $\gamma^{\prime}$ are homotopic relative endpoints. That is, assume there exists a continuous map $H:[0,1] \times[0,1] \rightarrow \mathfrak{M}$ with

$$
H(0, t)=\gamma(t), H(1, t)=\gamma^{\prime}(t), H(s, 0)=x \text { and } H(s, 1)=y \quad \text { for all } 0 \leq s \leq 1
$$

Then there exists partitions $0=s_{0}<s_{1}<\cdots<s_{\beta}=1$ and $0=t_{0}<t_{1}<\cdots<t_{\alpha}=1$ such that for each pair of indices $0 \leq j<\beta$ and $0 \leq k<\alpha$, there is an index $1 \leq i(j, k) \leq \nu$ such that

$$
H\left(\left[s_{j}, s_{j+1}\right] \times\left[t_{k}, t_{k+1}\right]\right) \subset D_{\mathcal{F}}\left(H\left(s_{j}, t_{k}\right), \epsilon_{\mathcal{U}}^{\mathcal{F}}\right) \subset U_{i(j, k)}
$$

A standard argument then yields the following basic fact about holonomy maps.

LEMMA 2.8. Let $\gamma, \gamma^{\prime}:[0,1] \rightarrow \mathfrak{M}$ be paths with $x=\gamma(0)=\gamma^{\prime}(0)$ and $y=\gamma(1)=\gamma^{\prime}(1)$, and suppose they are homotopic relative endpoints. Then the induced holonomy maps $h_{\gamma}$ and $h_{\gamma^{\prime}}$ agree on an open neighborhood of $\xi_{0}=\pi_{i_{0}}(x)$.

Next consider the groupoid formed by germs of maps in $\mathcal{G}_{\mathcal{F}}$. Let $U, U^{\prime}, V, V^{\prime} \subset \mathfrak{X}$ be open subsets with $w \in U \cap U^{\prime}$. Given homeomorphisms $h: U \rightarrow V$ and $h^{\prime}: U^{\prime} \rightarrow V^{\prime}$ with $h(w)=h^{\prime}(w)$, then $h$ and $h^{\prime}$ have the same germ at $w$, and write $h \sim_{w} h^{\prime}$, if there exists an open neighborhood $w \in W \subset U \cap U^{\prime}$ such that $h\left|W=h^{\prime}\right| W$. Note that $\sim_{w}$ defines an equivalence relation.

DEFINITION 2.9. The germ of $h$ at $w$ is the equivalence class $[h]_{w}$ under the relation $\sim_{w}$. The map $h: U \rightarrow V$ is called a representative of $[h]_{w}$. The point $w$ is called the source of $[h]_{w}$ and denoted $s\left([h]_{w}\right)$, while $w^{\prime}=h(w)$ is called the range of $[h]_{w}$ and denoted $r\left([h]_{w}\right)$.

The collection of all such germs $[h]_{w}$ for $h \in \mathcal{G}_{\mathcal{F}}$ and $w \in \operatorname{Dom}(h)$, forms the holonomy groupoid $\Gamma_{\mathcal{F}}$, which has the natural topology associated to sheaves of maps over $\mathcal{X}$. Let $\mathcal{R}_{\mathcal{F}} \subset \mathfrak{X} \times \mathfrak{X}$ denote the equivalence relation on $\mathfrak{X}$ induced by $\mathcal{F}$, where $\left(w, w^{\prime}\right) \in \mathcal{R}_{\mathcal{F}}$ if and only if $w, w^{\prime}$ correspond to points on the same leaf of $\mathcal{F}$. The product map $s \times r: \Gamma_{\mathcal{F}} \rightarrow \mathcal{R}_{\mathcal{F}}$ is étale; that is, the map is a local homeomorphism with discrete fibers. These notions were introduced by Haefliger for foliations [61, 62], and naturally extend to the case of matchbox manifolds.

We introduce a convenient notation for elements of $\Gamma_{\mathcal{F}}$. Let $\left(w, w^{\prime}\right) \in \mathcal{R}_{\mathcal{F}}$, and let $\gamma$ denote a path from $x=\tau(w)$ to $y=\tau\left(w^{\prime}\right)$. We may assume that $\gamma$ is a geodesic for the leafwise metric, and let $\left[h_{\gamma}\right]_{w}$ (or sometimes just $\gamma_{w}$ ) denote the germ at $w$ of the holonomy map defined by $\gamma$.

It follows that there is a well-defined surjective homomorphism, the holonomy map,

$$
h_{\mathcal{F}, x}: \pi_{1}\left(L_{x}, x\right) \rightarrow \Gamma_{w}^{w} \equiv\left\{[g]_{w} \in \Gamma_{\mathcal{F}} \mid r\left([g]_{w}\right)=w\right\}
$$

Moreover, if $y, z \in L$ then the homomorphism $h_{\mathcal{F}, y}$ is conjugate (by an element of $\mathcal{G}_{\mathcal{F}}$ ) to the homomorphism $h_{\mathcal{F}, z}$. A leaf $L$ is said to have non-trivial germinal holonomy if for some $y \in L$, the homomorphism $h_{\mathcal{F}, y}$ is non-trivial. If the homomorphism $h_{\mathcal{F}, y}$ is trivial, then we say that $L_{y}$ is a leaf without holonomy. This property depends only on $L$, and not the choice of $y \in L$. 
LEMMA 2.10. Given a path $\gamma:[0,1] \rightarrow \mathfrak{M}$ with $x=\gamma(0)$ and $y=\gamma(1)$. Suppose that $L_{x}$ is a leaf without holonomy. Then there exists a leafwise geodesic segment $\gamma^{\prime}:[0,1] \rightarrow \mathfrak{M}$ with $x=\gamma^{\prime}(0)$ and $y=\gamma^{\prime}(1)$, such that $\left\|\gamma^{\prime}\right\|=d_{\mathcal{F}}(x, y)$, and $h_{\gamma}$ and $h_{\gamma^{\prime}}$ agree on an open neighborhood of $\xi_{0}$.

Proof. The leaf $L_{x}$ containing $x$ is a complete Riemannian manifold, so there exists a geodesic segment $\gamma^{\prime}$ which is length minimizing between $x$ and $y$. Then the holonomy maps $h_{\gamma}$ and $h_{\gamma^{\prime}}$ agree on an open neighborhood of $\xi_{0}=\pi_{i_{0}}(x)$ by the definition of germinal holonomy.

Next, we introduce the filtrations of $\mathcal{G}_{\mathcal{F}}^{*}$ by word length, and of $\Gamma_{\mathcal{F}}$ by path length, then derive estimates comparing these notions of length.

For $\alpha \geq 1$, let $\mathcal{G}_{\mathcal{F}}^{(\alpha)}$ be the collection of holonomy homeomorphisms $h_{\mathcal{I}} \mid U \in \mathcal{G}_{\mathcal{F}}^{*}$ determined by admissible paths $\mathcal{I}=\left(i_{0}, \ldots, i_{k}\right)$ such that $k \leq \alpha$ and $U \subset \operatorname{Dom}\left(h_{\mathcal{I}}\right)$ is open. For each $\alpha$, let $C(\alpha)$ denote the number of admissible sequences of length at most $\alpha$. As there are at most $\nu^{2}$ admissible pairs $(i, j)$, we have the basic estimate that $C(\alpha) \leq \nu^{2 \alpha}$. This upper bound estimate grows exponentially with $\alpha$, though the exact growth rate of $C(\alpha)$ may be much less.

For each $g \in \mathcal{G}_{\mathcal{F}}^{*}$ there is some $\alpha$ such that $g \in \mathcal{G}_{\mathcal{F}}^{(\alpha)}$. Let $\|g\|$ denote the least such $\alpha$, which is called the word length of $g$. Note that $\mathcal{G}_{\mathcal{F}}^{(1)}$ generates $\mathcal{G}_{\mathcal{F}}^{*}$.

We use the word length on $\mathcal{G}_{\mathcal{F}}^{*}$ to define the word length on $\Gamma_{\mathcal{F}}$, where for $\gamma_{w} \in \Gamma_{\mathcal{F}}$, set

$$
\left\|\gamma_{w}\right\|=\min \left\{\|g\| \mid[g]_{w}=\gamma_{w} \text { for } g \in \mathcal{G}_{\mathcal{F}}^{*}\right\} .
$$

Introduce the path length of $\gamma_{w} \in \Gamma_{\mathcal{F}}$, by considering the infimum of the lengths $\left\|\gamma^{\prime}\right\|$ for all piecewise smooth curves $\gamma^{\prime}$ for which $\gamma_{w}^{\prime}=\gamma_{w}$. That is,

$$
\ell\left(\gamma_{w}\right)=\inf \left\{\left\|\gamma^{\prime}\right\| \mid \gamma_{w}^{\prime}=\gamma_{w}\right\} .
$$

Note that if $L_{w}$ is a leaf without holonomy, set $x=\tau(w)$ and $y=\tau\left(w^{\prime}\right)$, then Lemma 2.10 implies that $\ell\left(\gamma_{w}\right)=d_{\mathcal{F}}(x, y)$. This yields a fundamental estimate, whose proof can be found in [40]:

LEMMA 2.11. Let $[g]_{w} \in \Gamma_{\mathcal{F}}$ where $w$ corresponds to a leaf without holonomy. Then

$$
d_{\mathcal{F}}(x, y) / 2 \delta_{\mathcal{U}}^{\mathcal{F}} \leq\left\|[g]_{w}\right\| \leq 1+d_{\mathcal{F}}(x, y) / \epsilon_{\mathcal{U}}^{\mathcal{F}}
$$

\section{Pseudogroup Dynamics}

In this section, we consider some aspects of the topological dynamics of pseudogroups, which are useful for obtaining dynamical invariants for the pseudogroup $\mathcal{G}_{\mathfrak{X}}$ associated to a matchbox manifold. The sources [26, 78, 147] give more detailed discussions.

The study of the dynamics of a pseudogroup $\mathcal{G}_{\mathfrak{X}}$ acting on $\mathfrak{X}$ is a generalization of the study of continuous actions of finitely-generated groups on Cantor sets, though it differs in some fundamental ways. For a group action, each $\gamma \in \Gamma$ defines a homeomorphism $h_{\gamma}: \mathfrak{X} \rightarrow \mathfrak{X}$. For a pseudogroup action, given $g \in \mathcal{G}_{\mathfrak{X}}$ and $w \in \operatorname{Dom}(g)$, there is some clopen neighborhood $w \in U \subset \operatorname{Dom}(g)$ for which $g\left|U=h_{\mathcal{I}}\right| U$ where $\mathcal{I}$ is admissible sequence with $w \in \operatorname{Dom}\left(h_{\mathcal{I}}\right)$. By the definition of a pseudogroup, every $g \in \mathcal{G}_{\mathfrak{X}}$ is the "union" of such maps, and the dynamical properties of the action may reflect the fact that the domains of the actions are not all of $\mathfrak{X}$.

We first recall some basic definitions.

DEFINITION 3.1. A pseudogroup $\mathcal{G}_{\mathfrak{X}}$ acting on a Cantor set $\mathfrak{X}$ is compactly generated, if there exists two collections of clopen subsets, $\left\{U_{1}, \ldots, U_{k}\right\}$ and $\left\{V_{1}, \ldots, V_{k}\right\}$ of $\mathfrak{X}$, and homeomorphisms $\left\{h_{i}: U_{i} \rightarrow V_{i} \mid 1 \leq i \leq k\right\}$ which generate all elements of $\mathcal{G}_{\mathfrak{X}}$. The collection of maps $\mathcal{G}_{\mathfrak{X}}^{*}$ is defined to be all compositions of the generators on the maximal domains for which the composition is defined.

Let $d_{\mathfrak{X}}$ be a metric on $\mathfrak{X}$ which defines the topology on the space. 
DEFINITION 3.2. The action of a compactly generated pseudogroup $\mathcal{G}_{\mathfrak{X}}$ on $\mathfrak{X}$ is expansive, or more properly $\epsilon$-expansive, if there exists $\epsilon>0$ such that for all $w, w^{\prime} \in \mathfrak{X}$, there exists $g \in \mathcal{G}_{\mathfrak{X}}^{*}$ with $w, w^{\prime} \in D(g)$ such that $d_{\mathfrak{X}}\left(g(w), g\left(w^{\prime}\right)\right) \geq \epsilon$.

DEFINITION 3.3. The action of a compactly generated pseudogroup $\mathcal{G}_{\mathfrak{X}}$ on $\mathfrak{X}$ is equicontinuous if for all $\epsilon>0$, there exists $\delta>0$ such that for all $g \in \mathcal{G}_{\mathfrak{X}}^{*}$, if $w, w^{\prime} \in D(g)$ and $d_{\mathfrak{X}}\left(w, w^{\prime}\right)<\delta$, then $d_{\mathfrak{X}}\left(g(w), g\left(w^{\prime}\right)\right)<\epsilon$. Thus, $\mathcal{G}_{\mathfrak{X}}^{*}$ is equicontinuous as a family of local group actions.

The geometric entropy for pseudogroup actions, introduced by Ghys, Langevin and Walczak [59], gives a measure of the "exponential complexity" of the orbits of the action. See also the discussion of entropy for pseudogroup actions in Candel and Conlon [26, §13.2B], and in Walczak [147.

The key idea is the notion of $\epsilon$-separated sets, due to Bowen [23. Let $\epsilon>0$ and $\ell>0$. A subset $\mathcal{E} \subset \mathfrak{X}$ is said to be $\left(d_{\mathfrak{X}}, \epsilon, \ell\right)$-separated if for all $w, w^{\prime} \in \mathcal{E} \cap \mathfrak{X}_{i}$ there exists $g \in \mathcal{G}_{\mathfrak{X}}^{*}$ with $w, w^{\prime} \in \operatorname{Dom}(g) \subset \mathfrak{X}_{i}$, and $\|g\|_{w} \leq \ell$ so that $d_{\mathfrak{X}}\left(g(w), g\left(w^{\prime}\right)\right) \geq \epsilon$. If $w \in \mathfrak{X}_{i}$ and $w^{\prime} \in \mathfrak{X}_{j}$ for $i \neq j$ then they are $(\epsilon, \ell)$-separated by default. The "expansion growth function" counts the maximum of this quantity:

$$
h\left(\mathcal{G}_{\mathfrak{X}}, d_{\mathfrak{X}}, \epsilon, \ell\right)=\max \left\{\# \mathcal{E} \mid \mathcal{E} \subset \mathfrak{X} \text { is }\left(d_{\mathfrak{X}}, \epsilon, \ell\right) \text {-separated }\right\}
$$

The entropy is then defined to be the exponential growth type of the expansion growth function:

$$
h\left(\mathcal{G}_{\mathfrak{X}}, d_{\mathfrak{X}}, \epsilon\right)=\limsup _{\ell \rightarrow \infty} \ln \left\{h\left(\mathcal{G}_{\mathfrak{X}}, d_{\mathfrak{X}}, \epsilon, \ell\right)\right\} / \ell \quad, \quad h\left(\mathcal{G}_{\mathfrak{X}}, d_{\mathfrak{X}}\right)=\lim _{\epsilon \rightarrow 0} h\left(\mathcal{G}_{\mathfrak{X}}, d_{\mathfrak{X}}, \epsilon\right)
$$

Note that the quantity $h\left(\mathcal{G}_{\mathfrak{X}}, d_{\mathfrak{X}}\right) \geq 0$, and it may take the value $h\left(\mathcal{G}_{\mathfrak{X}}, d_{\mathfrak{X}}\right)=\infty$.

We recall two key properties of pseudogroup entropy. The first property follows directly from the definition of entropy.

PROPOSITION 3.4 (Proposition 2.6, [59]). Let $\mathcal{G}_{\mathfrak{X}}$ be a compactly generated pseudogroup, acting on the compact space $\mathfrak{X}$ with the metric $d_{\mathfrak{X}}$. Then the geometric entropy $h\left(\mathcal{G}_{\mathfrak{X}}, d_{\mathfrak{X}}\right)$ is independent of the choice of metric $d_{\mathfrak{X}}$.

The second property is an exercise using standard properties of the pseudogroup length function.

PROPOSITION 3.5 (Exercise 13.2.21, [26]). Let $\mathcal{G}_{\mathfrak{X}}$ be a compactly generated pseudogroup, acting on a compact space $\mathfrak{X}$ with the metric $d_{\mathfrak{X}}$. Then the property that $h\left(\mathcal{G}_{\mathfrak{X}}, d_{\mathfrak{X}}\right)$ is either zero, finite, or infinite, is independent of the choice of generating set for $\mathcal{G}_{\mathfrak{X}}$.

\section{LiPSCHITZ FOLIATIONS AND GEOMETRY}

In this section, we define the Lipschitz property for matchbox manifolds $\mathfrak{M}$. The basic result is that if $\mathfrak{M}$ is homeomorphic to an exceptional minimal set in a $C^{1}$-foliation, then its transversal space $\mathfrak{X}$ has a metric for which the induced pseudogroup $\mathcal{G}_{\mathfrak{X}}$ is Lipschitz.

It is a standard fact that there is a unique Cantor set, up to homeomorphism. That is, any two compact, perfect, totally disconnected and non-empty sets are homeomorphic. See [113, Chapter 12] for a proof and discussion of this result. In particular, for a given Cantor set $\mathfrak{X}$, any non-empty clopen subset $U \subset \mathfrak{X}$ is homeomorphic to $\mathfrak{X}$.

Two metrics $d_{\mathfrak{X}}$ and $d_{\mathfrak{X}}^{\prime}$ are Lipschitz equivalent if for some $C \geq 1$, they satisfy the condition:

$$
C^{-1} \cdot d_{\mathfrak{X}}(x, y) \leq d_{\mathfrak{X}}^{\prime}(x, y) \leq C \cdot d_{\mathfrak{X}}(x, y) \text { for all } x, y \in \mathfrak{X}
$$

On the other hand, there are many possible metrics on $\mathfrak{X}$ which are compatible with its topology, and need not be Lipschitz equivalent. The study of the Lipschitz geometry of the pair $\left(\mathfrak{X}, d_{\mathfrak{X}}\right)$ investigates the geometric properties common to all metrics in the Lipschitz class of the given metric $d_{\mathfrak{X}}$. Problem 1.4 can be rephrased as asking for characterizations of the transverse Lipschitz geometry of exceptional minimal sets. 
We next consider the Lipschitz property of matchbox manifolds. The choice of a regular foliated covering $\left\{\varphi_{i}: \bar{U}_{i} \rightarrow[-1,1]^{n} \times \mathfrak{X}_{i} \mid 1 \leq i \leq \nu\right\}$ for the matchbox manifold $\mathfrak{M}$, as in Proposition 2.6. yields the pseudogroup $\mathcal{G}_{\mathfrak{X}}$ which acts via homeomorphisms on the transversal space $\mathfrak{X}$ to $\mathcal{F}$.

DEFINITION 4.1. The action of a compactly generated pseudogroup $\mathcal{G}_{\mathfrak{X}}$ is C-Lipschitz with respect to $d_{\mathfrak{X}}$, if there exists a generating set $\left\{h_{i}: U_{i} \rightarrow V_{i} \mid 1 \leq i \leq k\right\}$ as in Definition 3.1, and $C \geq 1$, such that for each $1 \leq i \leq k$ and for all $w, w^{\prime} \in U_{i}$ we have

$$
C^{-1} \cdot d_{\mathfrak{X}}\left(w, w^{\prime}\right) \leq d_{\mathfrak{X}}\left(h_{i}(w), h_{i}\left(w^{\prime}\right)\right) \leq C \cdot d_{\mathfrak{X}}\left(w, w^{\prime}\right) .
$$

The condition (11) is equivalent to saying that $\mathcal{G}_{\mathfrak{X}}$ is generated by bi-Lipschitz homeomorphisms, though we use the notation Lipschitz for the action of the pseudogroup $\mathcal{G}_{\mathfrak{X}}$.

Recall that $\tau: \mathfrak{X} \rightarrow \mathcal{T} \subset \mathfrak{M}$ is the transversal to $\mathcal{F}$ associated to a regular covering of $\mathfrak{M}$. Let $d_{\mathfrak{X}}$ be the metric induced on $\mathfrak{X}$ by the restriction of $d_{\mathfrak{M}}$ on $\mathfrak{M}$ to the image of $\tau: \mathfrak{X} \rightarrow \mathcal{T}$.

The claim of the following result is intuitively clear, but its proof requires care with the subtleties of working with foliation charts that have totally disconnected transversals.

PROPOSITION 4.2. Let $\mathfrak{M}$ be a minimal matchbox manifold, and $M$ a smooth Riemannian manifold with a $C^{1}$-foliation $\mathcal{F}_{M}$, and $\mathcal{Z} \subset M$ an exceptional minimal set for $\mathcal{F}_{M}$. Suppose there exists a homeomorphism $f: \mathfrak{M} \rightarrow \mathcal{Z}$, then there exists a metric $d_{\mathfrak{X}}$ on $\mathfrak{X}$ such that the action of the holonomy pseudogroup $\mathcal{G}_{\mathfrak{X}}$ on $\mathfrak{X}$ is Lipschitz.

Proof. The map $f$ maps leaves of $\mathcal{F}$ to leaves of $\mathcal{F}_{M}$ by Lemma 2.7 and as $f$ is a homeomorphism onto its image, this implies the restriction of $f$ to a leaf $L$ of $\mathcal{F}$ is a homeomorphism onto a leaf $\mathcal{L}$ of $\mathcal{F}_{M}$, in the restricted topology on $\mathcal{Z}$.

Choose a good covering $\left\{\phi_{\alpha}: V_{\alpha} \rightarrow(-1,1)^{n} \times(-1,1)^{q} \mid 1 \leq \alpha \leq k\right\}$ for the foliation $\mathcal{F}$ of $M$, as in [26], where $n$ is the leaf dimension of $\mathcal{F}$, and $q$ is the codimension of $\mathcal{F}$ in $M$. Set $T_{\alpha}=$ $\phi_{\alpha}^{-1}\left(\{0\} \times(-1,1)^{q}\right)$, then the union $T=T_{1} \cup \cdots \cup T_{k}$ is a complete transversal for $\mathcal{F}$. We can assume without loss of generality that the closures of the transversals are disjoint. The Riemannian metric on $T M$ restricts to a Riemannian metric on each $T_{\alpha}$ and thus defines a path-length metric denoted by $d_{T_{\alpha}}$ on each submanifold $T_{\alpha} \subset M$. Extend the metrics on each $T_{\alpha}$ to a metric $d_{T}$ on $T$, by declaring $d_{T}(u, v)=1$ if $u \in T_{\alpha}$ and $v \in T_{\beta}$ for $\alpha \neq \beta$.

Recall that for $(\alpha, \beta)$ admissible, the overlap of plaques in the charts $V_{\alpha}$ and $V_{\beta}$ defines the holonomy map $g_{\alpha, \beta}$. The assumption that $\mathcal{F}$ is a $C^{1}$-foliation implies that $g_{\alpha, \beta}$ is a $C^{1}$-map from an open subset of $T_{\alpha}$ to an open set of $T_{\beta}$. For each $u \in \operatorname{Dom}\left(g_{\alpha, \beta}\right)$, let $D_{u}\left(g_{\alpha, \beta}\right)$ denote the matrix of differentials for $g_{\alpha, \beta}$ at $u \in \operatorname{Dom}\left(g_{\alpha, \beta}\right)$, with respect to the framing of the tangent spaces to the sections $T_{\alpha}$ induced by the coordinate charts. Let $\left\|D_{u}\left(g_{\alpha, \beta}\right)\right\|$ denote the matrix sup-norm of $D_{u}\left(g_{\alpha, \beta}\right)$ with respect to the Riemannian metric induced on the sections. The assumption that we have a good covering implies that the maps $g_{\alpha, \beta}$ admit continuous $C^{1}$-extensions, so the norms $\left\|D_{u}\left(g_{\alpha, \beta}\right)\right\|$ have uniform upper bounds for all admissible pairs $(\alpha, \beta)$ and all $u \in \operatorname{Dom}\left(g_{\alpha, \beta}\right)$. Define:

$$
C_{\mathcal{F}}^{\prime}=\max \left\{\left\|D_{u}\left(g_{\alpha, \beta}\right)\right\| \mid(\alpha, \beta) \text { admissible }, u \in \operatorname{Dom}\left(g_{\alpha, \beta}\right)\right\}<\infty
$$

It follows that the pseudogroup for $\mathcal{F}$ defined by the maps $\left\{g_{\alpha, \beta} \mid(\alpha, \beta)\right.$ admissible $\}$ is $C_{\mathcal{F}}^{\prime}$-Lipschitz.

Recall that $\mathcal{T}_{i} \subset \mathfrak{M}$, for $1 \leq i \leq \nu$, are the Cantor transversals to $\mathfrak{M}$ defined by a good covering for $\mathfrak{M}$, as in Definition 2.1. For each $x \in \mathcal{T}_{i}$ there exists $1 \leq \alpha \leq k$ with $f(x) \in V_{\alpha}$, and thus a clopen neighborhood $W(i, x, \alpha) \subset \mathcal{T}_{i}$ for which $f(W(i, x, \alpha)) \subset V_{\alpha}$. If $W(i, x, \alpha)$ is sufficiently small, then the plaque projection of the image, $\pi_{\alpha}: f(W(i, x, \alpha)) \rightarrow T_{\alpha}$, is a homeomorphism onto its image, and so the metric $d_{T_{\alpha}}$ on $T_{\alpha}$ induces a metric on $W(i, x, \alpha)$. As each $\mathcal{T}_{i}$ is compact, we can choose a finite covering $\left\{W_{k}\right\}$ of the union $\mathcal{T}=\mathcal{T}_{1} \cup \cdots \mathcal{T}_{\nu}$ where each $W_{k}=W(i, x, \alpha)$ for appropriate $(i, x, \alpha)$. It may happen that for $x, y \in W_{k}$ there is an admissible pair $(i, j)$ for the covering of $\mathfrak{M}$ such that $f\left(h_{i, j}(x)\right)$ and $f\left(h_{i, j}(y)\right)$ are not contained in the same foliation chart $V_{\ell}$. However, as there are only a finite number of admissible pairs $(i, j)$ for the covering of $\mathfrak{M}$ by foliation charts, we can refine the finite clopen covering $\left\{W_{k}\right\}$ of $\mathcal{T}$, so that this condition is satisfied. Then for each $W_{k}$ and $x \in W_{k}$ there is an index $i_{\alpha}$ such that $f(x) \in V_{\alpha}$ and $\pi_{\alpha}(f(x)) \in T_{\alpha}$. 
We then obtain a metric $d_{\mathcal{T}}$ on $\mathcal{T}$ by setting,

$$
d_{\mathcal{T}}(x, y)=d_{T_{\alpha}}\left(\pi_{\alpha}(f(x)), \pi_{\alpha}(f(y))\right) \quad \text { if } \quad x, y \in W_{k},
$$

and $d_{\mathcal{T}}(x, y)=1$ otherwise. The metric $d_{\mathcal{T}}$ induces a metric on $\mathfrak{X}$, denoted by $d_{\mathfrak{X}}$. We claim there exists $C_{\mathcal{F}} \geq 1$ such that the action of $\mathcal{G}_{\mathfrak{X}}$ on $\mathfrak{X}$ is $C_{\mathcal{F}}$-Lipschitz for $d_{\mathfrak{X}}$ and the generating set $\left\{h_{i, j} \mid(i, j)\right.$ admissible $\}$.

Suppose that $x, y \in W_{k}$, then $f\left(h_{i, j}(x)\right)$ and $f\left(h_{i, j}(y)\right)$ are contained in the same foliation chart $V_{\ell}$ by construction. Note that $x$ and $h_{i, j}(x)$ are contained in the same leaf of $\mathcal{F}$ so their images $f(x)$ and $f\left(h_{i, j}(x)\right)$ are contained in the same leaf of $\mathcal{F}$. Thus, there is a plaque chain of length at most $\lambda_{f, x}$ between these two points. The same holds for the point $y$, so there is a plaque-chain of length $\lambda_{f, y}$ between $f(y)$ and $f\left(h_{i, j}(y)\right)$. By the compactness of $\mathcal{T}$, there is a uniform upper bound $\lambda_{f}$ for all such pairs. Thus, by Lemma 4.3 we have the estimate for $x, y \in U_{k}$ with projections $w=\pi(x), w^{\prime}=\pi(y) \in \mathfrak{X}_{i}$, and $C_{\mathcal{F}}^{\prime \prime}=\left(C_{\mathcal{F}}^{\prime}\right)^{\lambda_{f}}$,

$$
\left(C_{\mathcal{F}}^{\prime \prime}\right)^{-1} \cdot d_{\mathfrak{X}}\left(w, w^{\prime}\right) \leq d_{\mathfrak{X}}\left(h_{i, j}(w), h_{i, j}\left(w^{\prime}\right)\right) \leq C_{\mathcal{F}}^{\prime \prime} \cdot d_{\mathfrak{X}}\left(w, w^{\prime}\right) .
$$

If $x, y$ do not belong to the same clopen set $W_{k}$, then $d_{\mathfrak{X}}\left(w, w^{\prime}\right)=1$ by definition, so their exists $C_{\mathcal{F}}^{\prime \prime \prime} \geq 1$ such that (13) holds for such pairs. Set $C_{\mathcal{F}}=\max \left\{C_{\mathcal{F}}^{\prime \prime}, C_{\mathcal{F}}^{\prime \prime \prime}\right\}$, and the claim follows.

We next give some properties of Lipschitz pseudogroups and their entropy. The following is an immediate consequence of the definitions.

LEMMA 4.3. Suppose that the action of $\mathcal{G}_{\mathfrak{X}}$ on $\mathfrak{X}$ is $C$-Lipschitz with respect to $d_{\mathfrak{X}}$. Then for all $g \in \mathcal{G}_{\mathfrak{X}}^{*}$ with word length $\|g\| \leq \alpha$, and $w, w^{\prime} \in \operatorname{Dom}(g)$ we have

$$
C^{-\alpha} \cdot d_{\mathfrak{X}}\left(w, w^{\prime}\right) \leq d_{\mathfrak{X}}\left(g(w), g\left(w^{\prime}\right)\right) \leq C^{\alpha} \cdot d_{\mathfrak{X}}\left(w, w^{\prime}\right) .
$$

We recall an application of Proposition 2.7 in [59], which gives conditions for $h\left(\mathcal{G}_{\mathfrak{X}}, d_{\mathfrak{X}}\right)<\infty$.

PROPOSITION 4.4. Let $\mathfrak{X} \subset \mathbb{R}^{q}$ be an embedded Cantor set, with metric $d_{\mathfrak{X}}$ obtained by the restriction of the standard metric on $\mathbb{R}^{q}$. Let $\mathcal{G}_{\mathfrak{X}}$ be a finitely generated pseudogroup, with generators $\left\{h_{i}: U_{i} \rightarrow V_{i} \mid 1 \leq i \leq k\right\}$, such that each $h_{i}$ is the restriction of a $C^{1}$ diffeomorphism defined on an open neighborhood in $\mathbb{R}^{q}$ of the compact set $U_{i}$. Then $\mathcal{G}_{\mathfrak{X}}$ with the metric $d_{\mathfrak{X}}$ is Lipshitz, and the geometric entropy $h\left(\mathcal{G}_{\mathfrak{X}}, d_{\mathfrak{X}}\right)<\infty$.

COROLLARY 4.5. Let $\mathfrak{M}$ be a matchbox manifold which embeds as an exceptional minimal set for $C^{1}$-foliation $\mathcal{F}$ on a compact smooth manifold $M$, as in Proposition 4.2. Then there is a transverse metric $d_{\mathfrak{X}}$ on $\mathfrak{X}$ such that $h\left(\mathcal{G}_{\mathfrak{X}}, d_{\mathfrak{X}}\right)<\infty$.

Proof. Let $d_{\mathfrak{X}}$ be the metric on $\mathfrak{X}$ constructed in the proof of Proposition 4.2. Then $\mathfrak{X}$ is covered by disjoint clopen sets for which $d_{\mathfrak{X}}$ is the pull-back of the metric on transversals to the foliation $\mathcal{F}$, so by Proposition 3.5 the entropy for the pseudogroup defined by $\mathcal{F}_{M}$ restricted to the image of $\mathfrak{M}$ and the entropy for $\mathcal{G}_{\mathfrak{X}}$ are either both zero, finite or infinite. Proposition 4.4 implies that both entropies are either zero or finite.

Note that by Proposition 3.4 , the entropy $h\left(\mathcal{G}_{\mathfrak{X}}, d_{\mathfrak{X}}\right)$ is independent of the choice of metric $d_{\mathfrak{X}}$ chosen for $\mathfrak{X}$, as long as it defines the topology for $\mathfrak{X}$. Thus by Corollary 4.5 we have:

COROLLARY 4.6. Let $\mathfrak{M}$ be a matchbox manifold with pseudogroup $\mathcal{G}_{\mathfrak{X}}$ for some regular covering of $\mathfrak{M}$. Suppose there exists a metric $d_{\mathfrak{X}}$ on $\mathfrak{X}$ for which $h\left(\mathcal{G}_{\mathfrak{X}}, d_{\mathfrak{X}}\right)=\infty$, then $\mathfrak{M}$ is not homeomorphic to an invariant set for any $C^{1}$-foliation.

It is well-known that entropy of a smooth non-singular flow on a compact manifold, when restricted to a compact invariant set $\mathcal{Z} \subset M$, is related to the Hausdorff dimension of $\mathcal{Z}$, as in [93, 94].

For a Lipschitz pseudogroup $h\left(\mathcal{G}_{\mathfrak{X}}, d_{\mathfrak{X}}\right)$, the box and Hausdorff dimension of $\mathfrak{X}$ with respect to $d_{\mathfrak{X}}$ are both well-defined, as in [52], and they do not depend on the Lipschitz equivalence class of the metric $d_{\mathfrak{X}}$. While there is no known direct relation between these dimensions and $h\left(\mathcal{G}_{\mathfrak{X}}, d_{\mathfrak{X}}\right)$, corresponding 
to the results for flows, there is a finiteness result based on a concept related to volume doubling for metric spaces (see [7, 20, 24]).

DEFINITION 4.7. A complete metric space $\left(\mathfrak{X}, d_{\mathfrak{X}}\right)$ has the doubling property, if there exists a constant $C>1$, such that for every $x \in \mathfrak{X}, r>0$, and integer $n>0$, the closed ball $B_{\mathfrak{X}}(x, r)$ of radius $r$ about $x$ admits a covering by $C^{n}$ balls of radius $r / 2^{n}$.

Note that if $\left(\mathfrak{X}, d_{\mathfrak{X}}\right)$ has the doubling property, then it has finite box dimension as well.

The proof of [26, Proposition 13.2.14] adapts directly to give:

PROPOSITION 4.8. If $h\left(\mathcal{G}_{\mathfrak{X}}, d_{\mathfrak{X}}\right)$ is a compactly generated Lipschitz pseudogroup such that $\left(\mathfrak{X}, d_{\mathfrak{X}}\right)$ has the doubling property, then $h\left(\mathcal{G}_{\mathfrak{X}}, d_{\mathfrak{X}}\right)<\infty$.

Thus, one approach to constructing matchbox manifolds which cannot embed into a smooth foliation, is to consider examples for which the transversal model space $\mathfrak{X}$ has infinite box dimension, for some metric. This will be discussed further in Section 8 .

The Hausdorff dimension of the transversal Cantor set to an exceptional minimal set for a $C^{1}$ foliation is well-studied, especially for foliations of codimension-one as in Cantwell and Conlon [30, Matsumoto [104, Gelfert and Rams 57, and Biś and Urbanski 18. Hausdorff dimension is also well-studied for Cantor sets defined by contracting Iterated Function Systems (or IFS's), and the more general class of self-similar fractals. For example, see the works of Rams and his coauthors in [46, 57, and the works of Rao, Ruan, Wang and Xi as in [122, 123], are closely related to the study of Lipschitz geometry of foliation minimal sets.

PROBLEM 4.9. Let $\mathfrak{M}$ be a Lipschitz matchbox manifold, with induced Lipschitz pseudogroup $\left(\mathcal{G}_{\mathfrak{X}}, d_{\mathfrak{X}}\right)$, and suppose $0<h\left(\mathcal{G}_{\mathfrak{X}}, d_{\mathfrak{X}}\right)<\infty$. Find properties of the Lipschitz geometry of $\mathfrak{X}$ which must be satisfied if the metric $d_{\mathfrak{X}}$ is induced by an embedding of $\mathfrak{M}$ into a $C^{r}$-foliation, for $r \geq 1$.

One can also define finer metric conditions on the action of a pseudogroup $\mathcal{G}_{\mathfrak{X}}$, such as the Zygmund condition used in [75] which can be used to define "quasi-conformal" properties of homeomorphisms, as in [56, 102, 118, 142, 144. The study of the Lipschitz properties of Gromov hyperbolic groups acting on their boundaries is a very well-developed subject; see for example [24, 85].

\section{EXAMPLES FROM FOLIATIONS}

In this section, we recall some examples of minimal matchbox manifolds which are realized as exceptional minimal sets in $C^{r}$-foliations, for $r \geq 1$. We first consider the case for foliations of codimension-one, for which the strongest results have been proven. The prototypical example is the well-known construction by Denjoy:

THEOREM 5.1 (Denjoy 48). There exist a $C^{1}$-diffeomorphism $f$ of the circle $\mathbb{S}^{1}$ with no fixed points, and with a non-empty wandering set $W$ such that the induced action of $f$ on the complement $\mathbf{K}=\mathbb{S}^{1}-W$ gives a minimal action, $\varphi: \mathbb{Z} \times \mathbf{K} \rightarrow \mathbf{K}$, called a Denjoy minimal system.

The $C^{1}$-hypotheses on the diffeomorphism $f$ is far from optimal. For example, McDuff [106] formulated a set of necessary and sufficient conditions on an embedded Cantor set $\mathbf{K} \subset \mathbb{S}^{1}$ so that it is an invariant set of a $C^{1+\alpha}$-diffeomorphism $f: \mathbb{S}^{1} \rightarrow \mathbb{S}^{1}$, for $0<\alpha<1$. Other optimal conditions on the derivative of a diffeomorphism $f: \mathbb{S}^{1} \rightarrow \mathbb{S}^{1}$ such that it admits a Cantor minimal set are discussed in $\mathrm{Hu}$ and Sullivan [71].

The Denjoy example played a fundamental role in the construction of counter-examples to the Seifert Conjecture, which enabled Schweitzer in 132 to construct the first $C^{1}$-examples of flows on 3-manifolds without periodic orbits. Schweitzer's construction embedded a suspension of the Denjoy minimal set as an isolated minimal set for a flow contained in a plug embedded in $\mathbb{R}^{3}$, and motivated Harrison's construction 63, 64 of a $C^{2+\alpha}$-flow in $\mathbb{R}^{3}$ with an isolated minimal limit set homeomorphic to a suspension of the Denjoy set, for $\alpha<1$. On the other hand, Knill constructed 
in [89] a smooth diffeomorphism in the 2-dimensional annulus with a minimal set homeomorphic to the Denjoy set, so the suspension of this diffeomorphism yields a codimension-2 smooth foliation defined by a flow, with a minimal set homeomorphic to the Denjoy minimal set in $\mathbb{T}^{2}$. Note that the periodic orbits for the Knill diffeomorphism contain the Denjoy set in its closure, so this example is not sufficient for constructing smooth counter-examples to the Seifert Conjecture. The Knill example illustrates that the degree of differentiability $r$ for a $C^{r}$-embedding of a Cantor minimal system may depend on the codimension, as well as the dynamical behavior of the action in open neighborhoods.

In some cases, there are analogs of the above results for the case of a finitely-generated group acting minimally on a Cantor set. For example, Pixton gave a generalization of the Denjoy construction:

THEOREM 5.2 (Pixton [120]). Suppose that $0<\alpha<1 /(n+1)$, then there exist a $C^{1+\alpha}$-action of $\mathbb{Z}^{n}$ on the circle $\mathbb{S}^{1}$ with no fixed points and with a non-empty wandering set $W$ so that the complement $\mathbf{K}=\mathbb{S}^{1}-W$ is a Cantor set which is minimal for the restricted action.

The Pixton-type examples have been further studied by Deroin, Kleptsyn and Navas in [49, and Kleptsyn and Navas in [88. Note that the suspension of such actions of $\mathbb{Z}^{n}$ on $\mathbb{S}^{1}$ yield foliations with exceptional minimal sets, whose leaves are diffeomorphic to $\mathbb{R}^{n}$.

Sacksteder proved in [127] that if $\mathcal{Z} \subset M$ is an exceptional minimal set for a codimension-one $C^{2}$ foliation $\mathcal{F}_{M}$ of a compact manifold $M$, then some leaf in $\mathcal{Z}$ must have an element of holonomy which is a transverse contraction, and thus cannot be of "Denjoy type". A special class of such examples, the Markov minimal sets, were studied by Hector [65, 66], Cantwell and Conlon [30, and Matsumoto [104. It remains an open problem to characterize the embeddings of Cantor minimal systems in $C^{r}$-foliations of codimension-one, for $r \geq 1$ (see [76]).

There are various constructions of $C^{r}$-foliations of codimension $q \geq 2$ with minimal sets which are matchbox manifold. Given a finitely-generated group $\Gamma$ and a $C^{r}$-action $\varphi: \Gamma \times N \rightarrow N$ on a compact manifold $N$ of dimension $q$, the suspension of the action (see [25]) yields a $C^{r}$-foliation of codimension- $q$. In general, it is impossible to determine if such an action $\varphi$ has an invariant Cantor set on which the action is minimal, except in very special cases. For example, consider a lattice subgroup $\Gamma \subset G$ of the rank one connected Lie group $G=S O(q, 1)$. The boundary at infinity for the associated symmetric space $\mathbb{H}^{q}=S O(q, 1) / O(q)$ is diffeomorphic to $\mathbb{S}^{q}$. If the group $\Gamma$ is a non-uniform lattice, then the action of $\Gamma$ on its limit set in $\mathbb{S}^{q}$ defines a minimal Cantor action, and the suspension of this action is a minimal matchbox manifold embedded in the smooth foliation associated to the action of $\Gamma$ on $\mathbb{S}^{q}$.

The Williams solenoids were introduced in the papers [148, 150]. Williams proved that for an Axiom A diffeomorphism $f: M \rightarrow M$ of a compact manifold $M$ with an expanding attractor $\Omega \subset M$, then $\Omega$ admits a stationary presentation, as defined in the next section, and so is homeomorphic to a generalized solenoid. The unstable manifolds for $f$ restricted to an open neighborhood $U$ of $\Omega$ form a $C^{0, \infty}$-foliation of $U$. That is, the foliation has $C^{0}$-pseudogroup maps, with smoothly embedded leaves, and $\Omega$ is the unique minimal set.

\section{Solenoids}

In this section, we describe the constructions of weak, normal and generalized solenoids, and recall some of their properties. We also give a construction of metrics on the transverse Cantor sets for which the holonomy action is by isometries, and hence equicontinuous. There are many open questions about when such examples can be realized as exceptional minimal sets for $C^{r}$-foliations.

A presentation is a collection $\mathcal{P}=\left\{p_{\ell+1}: M_{\ell+1} \rightarrow M_{\ell} \mid \ell \geq 0\right\}$, where each $M_{\ell}$ is a connected compact simplicial complex of dimension $n$, and each bonding map $p_{\ell+1}$ is a proper surjective map of simplicial complexes with discrete fibers. For $\ell \geq 0$ and $x \in M_{\ell}$, the set $\left\{p_{\ell+1}^{-1}(x)\right\} \subset M_{\ell+1}$ is compact and discrete, so the cardinality $\#\left\{p_{\ell+1}^{-1}(x)\right\}<\infty$. It need not be constant in $\ell$ or $x$. 
Associated to the presentation $\mathcal{P}$ is an inverse limit space, called a generalized solenoid,

$$
\mathcal{S}_{\mathcal{P}} \equiv \lim _{\longleftarrow}\left\{p_{\ell+1}: M_{\ell+1} \rightarrow M_{\ell}\right\} \subset \prod_{\ell \geq 0} M_{\ell} .
$$

By definition, for a sequence $\left\{x_{\ell} \in M_{\ell} \mid \ell \geq 0\right\}$, we have

$$
x=\left(x_{0}, x_{1}, \ldots\right) \in \mathcal{S}_{\mathcal{P}} \Longleftrightarrow p_{\ell}\left(x_{\ell}\right)=x_{\ell-1} \text { for all } \ell \geq 1 .
$$

The set $\mathcal{S}_{\mathcal{P}}$ is given the relative topology, induced from the product topology, so that $\mathcal{S}_{\mathcal{P}}$ is itself compact and connected.

For example, if $M_{\ell}=\mathbb{S}^{1}$ for each $\ell \geq 0$, and the map $p_{\ell}$ is a proper covering map of degree $m_{\ell}>1$ for $\ell \geq 1$, then $\mathcal{S}_{\mathcal{P}}$ is an example of a classic solenoid, discovered independently by van Dantzig [145] and Vietoris [146].

We say the presentation $\mathcal{P}$ is stationary if $M_{\ell}=M_{0}$ for all $\ell \geq 0$, and the bonding maps $p_{\ell}=p_{1}$ for all $\ell \geq 1$. A solenoid $\mathcal{S}_{\mathcal{P}}$ obtained from a stationary presentation $\mathcal{P}$ has a self-map $\sigma$ defined by the shift, $\sigma\left(x_{0}, x_{1}, \ldots\right)=\left(x_{1}, x_{2}, \ldots\right)$. The map $\sigma$ can be considered as a type of expanding map on $\mathcal{S}_{\mathcal{P}}$, though in fact it may be expanding only in some directions, as discussed in Section 3 of [17. By the work of Mouron 115, 116, these are the only examples of 1-dimensional solenoids with an expanding map. The case for expanding maps of generalized 1-dimensional solenoids is much richer, as described in the work of Williams [148, 149, which classifies the stationary inverse limits defined by expanding maps of branched 1-manifolds.

If $M_{\ell}$ is a compact manifold without boundary for each $\ell \geq 0$, and the map $p_{\ell}$ is a proper covering map of degree $m_{\ell}>1$ for $\ell \geq 1$, then $\mathcal{S}_{\mathcal{P}}$ is said to be a weak solenoid. This generalization of 1-dimensional solenoids was originally considered in the papers by McCord [105] and Schori [131]. In particular, McCord showed in [105] that $\mathcal{S}_{\mathcal{P}}$ has a local product structure, hence

PROPOSITION 6.1. Let $\mathcal{S}_{\mathcal{P}}$ be a weak solenoid, whose base space $M_{0}$ is a compact manifold of dimension $n \geq 1$. Then $\mathcal{S}_{\mathcal{P}}$ is a minimal matchbox manifold of dimension $n$.

Associated to a presentation $\mathcal{P}$ of compact manifolds is a sequence of proper surjective maps

$$
q_{\ell}=p_{1} \circ \cdots \circ p_{\ell-1} \circ p_{\ell}: M_{\ell} \rightarrow M_{0} .
$$

For each $\ell>1$, projection onto the $\ell$-th factor in the product $\prod_{\ell \geq 0} M_{\ell}$ in (15) yields a fibration map denoted by $\Pi_{\ell}: \mathcal{S}_{\mathcal{P}} \rightarrow M_{\ell}$, for which $\Pi_{0}=\Pi_{\ell} \circ q_{\ell}: \mathcal{S}_{\mathcal{P}} \rightarrow M_{0}$. A choice of a basepoint $x \in \mathcal{S}_{\mathcal{P}}$ gives basepoints $x_{\ell}=\Pi_{\ell}(x) \in M_{\ell}$, and we define $\mathcal{H}_{\ell}^{x}=\pi_{1}\left(M_{\ell}, x_{\ell}\right)$. Let $\mathfrak{X}_{x}=\Pi_{0}^{-1}(x)$ denote the fiber of $x$, which is Cantor set by the assumption on the cardinality of the fibers of each map $p_{\ell}$.

A presentation $\mathcal{P}$ is said to be normal if, given a basepoint $x \in \mathcal{S}_{\mathcal{P}}$, for each $\ell \geq 1$ the image subgroup of the map $\left(q_{\ell}\right)_{\#}: \mathcal{H}_{\ell}^{x} \longrightarrow \mathcal{H}_{0}^{x}$ is a normal subgroup. Then each quotient $G_{\ell}^{x}=\mathcal{H}_{0}^{x} / \mathcal{H}_{\ell}^{x}$ is finite group, and there are surjections $G_{\ell+1}^{x} \rightarrow G_{\ell}^{x}$. The fiber $\mathfrak{X}_{x}$ is then naturally identified with the Cantor group defined by the inverse limit,

$$
G_{\infty}^{x}=\lim _{\longleftarrow}\left\{p_{\ell+1}: G_{\ell+1}^{x} \rightarrow G_{\ell}^{x}\right\} \subset \prod_{\ell \geq 0} G_{\ell}^{x} .
$$

The fundamental group $\mathcal{H}_{0}^{x}$ acts on the fiber $G_{\infty}^{x}$ via the coordinate-wise multiplication on the product in (17). In the case of the Vietoris solenoid, where each map $p_{\ell}: \mathbb{S}^{1} \rightarrow \mathbb{S}^{1}$ is a double cover, the fiber $G_{\infty}^{x}$ is the dyadic group. More generally, a solenoid $\mathcal{S}_{\mathcal{P}}$ is said to be a normal (or McCord) solenoid if the tower of coverings in the presentation is normal, and thus the fiber over $x \in M_{0}$ of the map $\mathcal{S}_{\mathcal{P}} \rightarrow M_{0}$ is the Cantor group $G_{\infty}^{x}$.

LEMMA 6.2. Let $\mathcal{P}$ be a presentation of a weak solenoid $\mathcal{S}_{\mathcal{P}}$, choose a basepoint $x \in \mathcal{S}_{\mathcal{P}}$ and set $\mathfrak{X}_{x}=\Pi_{0}^{-1}(x)$, and recall that $\mathcal{H}_{0}^{x}=\pi_{1}\left(M_{0}, x_{0}\right)$. Then the left action of $\mathcal{H}_{0}^{x}$ on $\mathfrak{X}_{x}$ is minimal.

Proof. The left action of $\mathcal{H}_{0}^{x}$ on each quotient space $X_{\ell}=\mathcal{H}_{0}^{x} / \mathcal{H}_{\ell}^{x}$ is transitive, so the orbits are dense in the product topology for $\mathfrak{X}_{x}$. 
Let $\widetilde{M}_{0}$ denote the universal covering of the compact manifold $M_{0}$. Associated to the left action of $\mathcal{H}_{0}^{x}$ on $\mathfrak{X}_{x}$ is a suspension minimal matchbox manifold

$$
\mathfrak{M}=\widetilde{M}_{0} \times \mathfrak{X}_{x} /\left(y_{0} \cdot g^{-1}, x\right) \sim\left(y_{0}, g \cdot x\right) \quad \text { for } y_{0} \in \widetilde{M}_{0}, g \in \mathcal{H}_{0}^{x} .
$$

Given coverings $\pi^{\prime}: M^{\prime} \rightarrow M$ and $\pi^{\prime \prime}: M^{\prime \prime} \rightarrow M$, such that the subgroups

$$
\pi_{\#}^{\prime}\left(\pi_{1}\left(M^{\prime}, x^{\prime}\right)\right)=\pi_{\#}^{\prime \prime}\left(\pi_{1}\left(M^{\prime \prime}, x^{\prime \prime}\right)\right) \subset \pi_{1}(M, x),
$$

then there is a natural homeomorphism of coverings $M^{\prime} \cong M^{\prime \prime}$ which is defined using the path lifting property. From this, it easily follows (see [38]) that:

PROPOSITION 6.3. Let $\mathcal{S}_{\mathcal{P}}$ be a weak solenoid with base space $M_{0}$ where $M_{0}$ is a compact manifold of dimension $n \geq 1$. Then there is a foliated homeomorphism $\mathcal{S}_{\mathcal{P}} \cong \mathfrak{M}$.

COROLLARY 6.4. The homeomorphism type of a weak solenoid $\mathcal{S}_{\mathcal{P}}$ is completely determined by the base manifold $M_{0}$ and the descending chain of subgroups

$$
\mathcal{H}_{0}^{x} \supset \mathcal{H}_{1}^{x} \supset \mathcal{H}_{2}^{x} \supset \mathcal{H}_{3}^{x} \supset \cdots
$$

Note the intersection $\mathcal{H}_{\infty}^{x} \equiv \cap_{\ell \geq 1} \mathcal{H}_{2}^{x}$ is the fundamental group of the typical leaf of $\mathcal{P}$. If this intersection group is trivial, then all leaves of the foliation $\mathcal{F}$ for $\mathfrak{M} \cong \mathcal{S}_{\mathcal{P}}$ are isometric to the universal covering of the base manifold $M_{0}$.

The presentation $\mathcal{P}$ of an inverse limit $\mathcal{S}_{\mathcal{P}}$ can be used to construct a "natural" metric on the space, and which is well-adapted to Lipschitz maps between such spaces. This has been studied in detail in the works by Miyata and Watanabe [108, 109, 110, 111, 112. In the case of weak solenoids, this construction of natural metrics adapted to the resolution takes on a simplified form.

Let $\mathcal{S}_{\mathcal{P}}$ be a weak solenoid, with notations as above. Then each quotient $X_{\ell}=\mathcal{H}_{0}^{x} / \mathcal{H}_{\ell}^{x}$ is a finite set with a transitive left action of the fundamental group $\mathcal{H}_{0}^{x}$. Let $d_{\ell}$ denote the discrete metric on $X_{\ell}$, where $d_{\ell}(x, y)=1$ unless $x=y$, for $x, y \in X_{\ell}$. Observe that the left action of $\mathcal{H}_{0}^{x}$ acts by isometries for the metric $d_{\ell}$. Choose a positive series $\left\{a_{\ell} \mid a_{\ell}>0\right\}$ with total sum 1 , then define a metric on $\mathfrak{X}_{x}$ by setting, for $u, v \in \mathfrak{X}_{x}$ so $u=\left(x_{0}, u_{1}, u_{2}, \ldots\right)$ and $v=\left(x_{0}, v_{1}, v_{2}, \ldots\right)$,

$$
d_{\mathfrak{X}}(u, v)=a_{1} d_{1}\left(u_{1}, v_{1}\right)+a_{2} d_{2}\left(u_{2}, v_{2}\right)+\cdots
$$

Then $d_{\mathfrak{X}}$ is invariant under the action of $\mathcal{H}_{0}^{x}$, so the holonomy for the fibration $\Pi_{0}: \mathcal{S}_{\mathcal{P}} \rightarrow M_{0}$ acts by isometries for this metric on $\mathfrak{X}_{x}$.

It may happen that we have two presentations $\mathcal{P}$ and $\mathcal{P}^{\prime}$ over the same base manifold $M_{0}$ such that their inverse limits are homeomorphic as fibrations, $h: \mathcal{S}_{\mathcal{P}} \cong \mathcal{S}_{\mathcal{P}^{\prime}}$. However, the map $h$ need not be Lipschitz on fibers for the metrics associated to the presentations as above, as will be seen in the examples in Section 9 ,

The normal solenoids have a nice characterization among the matchbox manifolds. A continuum $\Omega$ is homogeneous if its group of homeomorphisms is transitive. That is, given any two points $x, y \in \mathfrak{M}$, there is a homeomorphism $h: \mathfrak{M} \rightarrow \mathfrak{M}$ such that $h(x)=y$. It was shown in 38, that:

THEOREM 6.5. Let $\mathfrak{M}$ be a homogeneous matchbox manifold. Then $\mathfrak{M}$ is homeomorphic to a normal solenoid $\mathcal{S}_{\mathcal{P}}$ as foliated spaces.

The normal solenoids are the analogs in codimension-zero foliation theory, for the transversely parallelizable (TP) equicontinuous foliations in a topological version of Molino's Theory for smooth foliations of manifolds [5. Note that all leaves in a normal solenoid are homeomorphic, as the spaces are homogeneous. In the case of weak solenoids, the leaves of $\mathcal{F}$ need not be homeomorphic, and the works [36, 47] give examples where the leaves of $\mathcal{F}$ have differing numbers of ends. There is no analog of this behavior in the context of smooth Riemannian foliations on manifolds.

Now consider a matchbox manifold $\mathfrak{M}$ of dimension $n$, but whose associated pseudogroup $\mathcal{G}_{\mathfrak{X}}$ is not equicontinuous. This type of matchbox manifold arises in the study of the tiling spaces associated to aperiodic tilings of $\mathbb{R}^{n}$ with finite local complexity, and also as foliation minimal sets. For example, the Hirsch examples in [68] (see also [17]) yield real analytic foliations of codimension-one with 
exceptional minimal sets and expansive holonomy pseudogroups. Also, the exceptional minimal sets for the Denjoy and Pixton examples discussed in Section 5 have the property that all of their leaves are diffeomorphic to $\mathbb{R}^{n}$, and so they are without leafwise holonomy, but the global holonomy pseudogroup $\mathcal{G}_{\mathfrak{X}}$ associated to them is not equicontinuous. It follows from the following result that each of their minimal sets admits a presentation of the form (15).

THEOREM 6.6 (40]). Let $\mathfrak{M}$ be a minimal matchbox manifold without germinal holonomy. Then there exists a presentation $\mathcal{P}$ by simplicial maps between compact branched manifolds, such that $\mathfrak{M}$ is homeomorphic to $\mathcal{S}_{\mathcal{P}}$ as foliated spaces.

COROLLARY 6.7. Let $\mathfrak{M}$ be an exceptional minimal set for a $C^{1}$-foliation $\mathcal{F}$ of a compact manifold $M$. If all leaves of $\mathcal{F} \mid \mathfrak{M}$ are simply connected, then there is a homeomorphism of $\mathfrak{M}$ with the inverse limit space $\mathcal{S}_{\mathcal{P}}$ defined by a presentation $\mathcal{P}$, given by simplicial maps between compact branched manifolds.

In the case of the Denjoy and Pixton examples given in Theorems 5.1 and 5.2, the geometry of their construction implies that the presentation $\mathcal{P}$ one obtains is stationary.

PROBLEM 6.8. Let $\mathfrak{M}$ be an exceptional minimal set for a $C^{r}$-foliation $\mathcal{F}$ of a compact manifold $M$, where $r \geq 1$, and assume that $\mathfrak{M}$ is without holonomy. Find conditions on the holonomy pseudogroup $\mathcal{G}_{\mathfrak{X}}$ for $\mathcal{F}$ which are sufficient to imply that $\mathfrak{M}$ admits a stationary presentation.

One approach to this problem, is to ask if the existence of approximations to the foliation $\mathcal{F}$ on $\mathfrak{M}$ by the compact branched manifolds $M_{\ell}=M_{0}$ in a stationary presentation $\mathcal{P}$, implies some form of "finiteness" for the holonomy maps of the pseudogroup $\mathcal{G}_{\mathfrak{X}}$. Such finiteness conditions may be derived, for example, from the induced action of the shift map $\sigma$ on the tower of maps in the presentation. Then one would try to "fill in" the approximations with a foliation on an open neighborhood. Such a result would be reminiscent of the approach to showing the vanishing of the Godbillon-Vey class by Duminy and Sergiescu in [51.

Theorem [6.6 is a generalization of a celebrated result by Anderson and Putnam in [6] for tiling spaces. Given a repetitive, aperiodic tiling of the Euclidean space $\mathbb{R}^{n}$ with finite local complexity, the associated tiling space $\Omega$ is defined as the closure of the set of translations by $\mathbb{R}^{n}$ of the given tiling, in an appropriate topology on the space of tilings on $\mathbb{R}^{n}$. The space $\Omega$ is a matchbox manifold in our sense, whose leaves are defined by a free action of $\mathbb{R}^{n}$ on $\Omega$ (for example, see [121, 128, 130.) A remarkable result in the theory of tilings of $\mathbb{R}^{n}$ is that the tiling space $\Omega$ admits a presentation as the inverse limit of a tower of branched flat manifolds [6, 129, 130, where the branched manifolds are the union of finite collections of tiles.

Other generalizations of the Anderson-Putnam theorem have been given. For example, the work of Benedetti and Gambaudo in [15] discusses constructing towers for special classes of matchbox manifolds with possibly non-trivial but finite holonomy, where the leaves are defined by a locally-free action of a connected Lie group $G$. Their work suggests what appears to be a difficult problem:

PROBLEM 6.9. Let $\mathfrak{M}$ be a minimal matchbox manifold with leaves having non-trivial holonomy. Show that $\mathfrak{M}$ is homeomorphic to an inverse limit $\mathcal{S}_{\mathcal{P}}$ for some modified notion of presentations by branched manifolds, which takes into account the leafwise holonomy groups.

Note that a solution to this problem would yield a presentation for an exceptional minimal set in a $C^{2}$-foliation of codimension-one, which by the results of Sacksteder in 127. always have leaves with holonomy. The existence of such a presentation would provide an alternate approach to the celebrated result of Duminy on the ends of leaves in exceptional minimal sets [31.

Theorem 5.8 in the paper [97] states a solution to Problem 6.9 though it seems that the claimed result conflicts with the results of [15] for a model of generalized tiling spaces defined by $G$-actions with non-trivial holonomy. Also, the results of Section 6 of the same paper conflict with other established results concerning weak solenoids. 
PROBLEM 6.10. Given a weak solenoid $\mathcal{S}_{\mathcal{P}}$ with presentation $\mathcal{P}$ and associated transverse metric given by (20), does there exists a Lipschitz embedding of $\mathcal{S}_{\mathcal{P}}$ as an exceptional minimal set for a $C^{r}$-foliation of a smooth manifold $M$ ?

The problem is of interest whether $M$ is assumed compact, or open without boundary, and for any $r \geq 1$. All known results are for the case where the base $M_{0}=\mathbb{T}^{n}$ is a torus, for $n \geq 1$. The examples of type DE (Derived from Expanders) described by Smale in [136, p. 788], constructs an embedding of the dyadic solenoid over $\mathbb{S}^{1}$ which is realised as a basic set for a smooth diffeomorphism and is an attractor. More general realizations of 1-dimensional solenoids as minimal sets for smooth flows were constructed for flows in the works by Gambaudo and Tresser [55, Gambaudo, Sullivan and Tresser [55], and Markus and Meyer [103]. The case when the base manifold $M_{0}=\mathbb{T}^{n}$ for $n \geq 2$ was studied by the author with Clark in [37, where it was shown that for every presentation $\mathcal{P}$ there exists a refinement $\mathcal{P}^{\prime}$ which can be realized in a $C^{r}$-foliation. That is, every topological type can be realized, though the metric induced on the inverse limit depends on the presentation $\mathcal{P}$.

All of the known examples of weak solenoids which embed as exceptional minimal sets for $\mathrm{C}^{2}$ foliations have abelian fundamental group $\mathcal{H}_{x}$ and so are consequently normal solenoids. It seems plausible, based on the proofs in [37, to conjecture that if a weak solenoid admits an embedding in a $C^{2}$-foliation, then it must be a normal solenoid with nilpotent covering groups. It also seems possible that an even stronger conclusion holds, that the covering groups for such a smoothly embedded solenoid must be abelian.

\section{Fusion of Cantor minimal systems}

There is a well-known method, called tubularization, of amalgamating the holonomy pseudogroups of two foliations $\mathcal{F}_{1}, \mathcal{F}_{2}$ of codimension-one with the same leaf dimension. We recall this method briefly, then introduce the analogue of this technique for minimal matchbox manifolds, to obtain the fusion of their holonomy pseudogroups.

Assume there are given two foliations say $\mathcal{F}_{1}$ and $\mathcal{F}_{2}$, on manifolds $M_{1}$ and $M_{2}$, of with leaf dimension $n$ and codimension-one. We assume that their normal bundles are oriented, and there are given smooth embeddings $\eta_{i}: \mathbb{S}^{1} \rightarrow M_{i}$ which are transverse to $\mathcal{F}_{i}$ for $i=1,2$. For $\epsilon>0$ small, let $\mathcal{E}\left(\eta_{i}, \epsilon\right) \subset M_{i}$ be the closed $\epsilon$-disk neighborhood of the image of the map $\eta_{i}$, where we assume $\epsilon>0$ is chosen so that $\mathcal{E}\left(\eta_{i}, \epsilon\right)$ is an embedded submanifold with boundary diffeomorphic to $\mathbb{T}^{2}$. Then the restriction of $\mathcal{F}_{i}$ to $\mathcal{E}\left(\eta_{i}, \epsilon\right)$ is a foliation whose leaves are closed 2-disks, and which are parametrized by $\mathbb{S}^{1}$ via the transversal $\eta_{i}$.

The choice of a diffeomorphism $\varphi: \mathbb{S}^{1} \rightarrow \mathbb{S}^{1}$ extends to give a foliated map $\widehat{\varphi}: \mathcal{E}\left(\eta_{1}, \epsilon\right) \rightarrow \mathcal{E}\left(\eta_{2}, \epsilon\right)$, which we use to identify the boundaries $\partial \mathcal{E}\left(\eta_{1}, \epsilon\right)$ and $\partial \mathcal{E}\left(\eta_{2}, \epsilon\right)$. Denote the resulting surgered manifold by $M=M_{1} \#_{\varphi} M_{2}$. Then $M$ has a foliation of codimension-one, whose foliation pseudogroup is the amalgamation, or "pseudogroup free product", of the pseudogroups for $\mathcal{F}_{1}$ and $\mathcal{F}_{2}$. This very useful construction has many applications $[25,26,65,92$.

For foliations with codimension $q>1$, the tubularization method is not so commonly used, as the existence of a compact manifold $N$ and embeddings $\eta_{i}: N \rightarrow M_{i}$ transverse to the given foliations is a highly exceptional condition to assume. The tubularization method is often replaced with the method of spinnable structures of Tamura [143], or the open book method as in [91, 151].

Next, we define the analog of tubularization for Cantor pseudogroups. We first describe this construction for group actions. Assume there are given actions $\varphi_{i}: \Gamma_{i} \times \mathbf{K}_{i} \rightarrow \mathbf{K}_{i}$ for $i=1,2$, of finitely generated groups $\Gamma_{i}$ on Cantor sets $\mathbf{K}_{i}$. Choose clopen subsets $V_{i} \subset \mathbf{K}_{i}$ and a homeomorphism $h: V_{1} \rightarrow V_{2}$. Define the Cantor set $\mathbf{K}=\mathbf{K}_{1} \#_{h} \mathbf{K}_{2}$ obtained from the disjoint union $\mathbf{K}_{1} \cup \mathbf{K}_{2}$ by identifying the clopen subsets $V_{1}$ and $V_{2}$ using the map $h$.

The action on $\mathbf{K}$ of $\gamma \in \Gamma_{1}$ is via $\varphi_{1}(\gamma)$ on $\mathbf{K}_{1}$, and acts as the identity on the complement $\mathbf{K}_{2}-V_{2}$. Analogously, the action of $\varphi_{2}$ extends to an action of the elements of $\Gamma_{2}$ on $\mathbf{K}$. This produces an 
action $\varphi$ of the free product $\Gamma_{1} * \Gamma_{2}$ on $\mathbf{K}$. Note that if $V_{1}=\mathbf{K}_{1}$ and $V_{2}=\mathbf{K}_{2}$ then this process is just combining the generators of $\varphi_{1}\left(\Gamma_{1}\right)$ with the conjugates by $h$ of the generators of $\varphi_{2}\left(\Gamma_{2}\right)$.

If each of the actions $\varphi_{i}$ is minimal, then the action of $\varphi$ on $\mathbf{K}$ is also minimal.

In the case where $\mathcal{G}_{\mathbf{K}_{1}}$ is a pseudogroup acting on $\mathbf{K}_{1}$ and $\mathcal{G}_{\mathbf{K}_{2}}$ is a pseudogroup acting on $\mathbf{K}_{2}$, then the amalgamation of their actions over a homeomorphism $h: V_{1} \rightarrow V_{2}$ is actually simpler, as there is no need to extend the domains of the local actions.

If the action $\varphi_{i}$ is realized as the holonomy of a suspension matchbox manifold $\mathfrak{M}_{i}$ as in (18), then the action of $\varphi$ is realized as the holonomy of a surgered matchbox manifold $\mathfrak{M}=\mathfrak{M}_{1} \#_{h} \mathfrak{M}_{2}$ constructed analogously to the method described above for codimension-one foliations. This construction is analogous to the construction of a new graph matchbox manifold, from two given graph matchbox manifolds, which was introduced by Lukina in [99] as part of her study of the dynamics of examples obtained by the Ghys-Kenyon construction. Lukina called this process "fusion", and we adopt the same terminology for the process described here.

DEFINITION 7.1. Let $\mathfrak{M}_{i}$ be minimal matchbox manifolds with transversals $\mathfrak{X}_{i}$ for $i=1,2$. Choose clopen subsets $V_{i} \subset \mathfrak{X}_{i}$ and a homeomorphism $h: V_{1} \rightarrow V_{2}$. Then the minimal matchbox manifold $\mathfrak{M}=\mathfrak{M}_{1} \#_{h} \mathfrak{M}_{2}$ is said to be the fusion of $\mathfrak{M}_{1}$ with $\mathfrak{M}_{2}$ over $h$.

The concept of fusion for matchbox manifolds illustrates some of their fundamental differences with smooth foliations. A clopen transversal for a smooth foliation must be a compact submanifold without boundary, which does not always exist, while the above fusion construction can always be defined, along with many variations of it. Here is an interesting basic question:

PROBLEM 7.2. How are the dynamical properties of a fusion $\mathfrak{M}=\mathfrak{M}_{1} \#_{h} \mathfrak{M}_{2}$ related to the dynamical properties of the factors $\mathfrak{M}_{1}$ and $\mathfrak{M}_{2}$ ? In particular, describe the geometric structure of the leaves in $\mathfrak{M}$, in terms of the structure of the leaves of the factors $\mathfrak{M}_{1}$ and $\mathfrak{M}_{2}$ and the fusion map $h: V_{1} \rightarrow V_{2}$ between transversals. Show that the theory of hierarchies for the leaves of graph matchbox manifolds in Lukina 99. also apply for fusion in the context of matchbox manifolds.

\section{NON-EMBEDDABLE MATCHBOX MANIFOLDS}

In this section, we construct examples of Lipschitz pseudogroups $\left(\mathcal{G}_{\mathfrak{X}}, d_{\mathfrak{X}}\right)$ which cannot arise from an embedding of a matchbox manifold into a $C^{1}$-foliation. All of the pseudogroups constructed can be realized as the holonomy of a matchbox manifold $\mathfrak{M}$, using the suspension construction described in [98. Thus, the resulting matchbox manifolds $\mathfrak{M}$ do not embed as closed invariant sets for any $C^{1}$-foliation. There are many variations on the constructions, which shows that there is a wide variety of non-embeddable matchbox manifolds.

The idea of the construction is to produce a Lipschitz pseudogroup $\mathcal{G}_{\mathfrak{X}}$ with infinite entropy, $h\left(\mathcal{G}_{\mathfrak{X}}, d_{\mathfrak{X}}\right)=\infty$, so that by Corollary 4.5 the associated suspension matchbox manifold is not homeomorphic to an exceptional minimal set. Achieving infinite entropy with Lipschitz generators for $\mathcal{G}_{\mathfrak{X}}$ requires that the space $\left(\mathfrak{X}, d_{\mathfrak{X}}\right)$ have infinite Hausdorff dimension. The first step then, is the construction of the model for the metric Cantor set $\left(\mathfrak{X}, d_{\mathfrak{X}}\right)$, which is based on the construction of graph matchbox manifolds, as introduced by Ghys in [60], and studied in [19, 96, 98, 100].

Let $\mathcal{T}$ be an infinite connected tree with bounded valence. The example that we consider here is the Cayley graph $T_{n}$ for the free group on $n$-generators, $\mathbb{F}_{n}=\mathbb{F} * \cdots * \mathbb{F}$, for $n \geq 2$. Choose a basepoint $e \in \mathcal{T}$. Each edge of $\mathcal{T}$ is homeomorphic to $[0,1]$ so inherits a metric from $\mathbb{R}$. Then give $\mathcal{T}$ the path length metric, and let $B_{\mathcal{T}}(x, n) \subset \mathcal{T}$ denote the closed ball of radius $n$ centered at $x \in \mathcal{T}$. Thus, if $x$ is a vertex of the tree, then $B_{\mathcal{T}}(x, n)$ is a connected subtree of $\mathcal{T}$.

We say that a subtree $T \subset \mathcal{T}$ has a dead end, if there is a vertex $x \in T$ which is contained in a unique edge. Let $X$ be the set of all connected subtrees of $\mathcal{T}$ which have no dead ends, and such that $e \in T$. Define the metric $d_{X}$ on $X$ by declaring that, for $T, T^{\prime} \in X$ then

$$
d_{X}\left(T, T^{\prime}\right) \leq 2^{-n} \Longleftrightarrow B_{\mathcal{T}}(x, n) \cap T=B_{\mathcal{T}}(x, n) \cap T^{\prime} .
$$


Let $\mathfrak{X}$ denote the closure of $X$ in this metric, then $\mathfrak{X}$ is a totally disconnected space. A point $z \in \mathfrak{X}$ is then a subtree of $\mathcal{T}$ which contains the basepoint $e$.

In the case where $\mathcal{T}_{n}$ is the Cayley graph of $\mathbb{F}_{n}$, we denote the closure of the space of subtrees of $\mathcal{T}_{n}$ as above by $\mathfrak{X}_{n}$. The "no dead end" assumption on the subtrees implies that $\mathfrak{X}_{n}$ has no isolated points, hence is a Cantor set. Let $d_{\mathfrak{X}_{n}}$ denote the induced metric on $\mathfrak{X}_{n}$. Then we have:

THEOREM 8.1 (Lukina [100]). For $n \geq 2$, the metric space $\left(\mathfrak{X}_{n}, d_{\mathfrak{X}_{n}}\right)$ has infinite Hausdorff dimension.

The translation action of $\mathbb{F}_{n}$ on $\mathcal{T}_{n}$ defines a pseudogroup $\mathcal{G}_{\mathfrak{X}_{n}}$ acting on $\mathfrak{X}_{n}$, where a word $\gamma \in \mathbb{F}^{n}$ acts on the pointed subtree $(T, e)$ if $\gamma \cdot e \in T$, so that $\left(\gamma^{-1} \cdot T, e\right) \in \mathfrak{X}_{n}$. This action is discussed further in [98, 100. In particular, the action is Lipschitz for the metric $d_{\mathfrak{X}_{n}}$, with constant $C=2$.

Lukina shows in 99] that there exists a dense orbit for this action, so the pseudogroup is transitive. However, the periodic orbits for the action of $\mathcal{G}_{\mathfrak{X}_{n}}$ are dense, so the action is not minimal.

The proof of Theorem 8.1 in [100] essentially shows the following, with the details given in [80]:

THEOREM 8.2. For $n \geq 2, h\left(\mathcal{G}_{\mathfrak{X}_{n}}, d_{\mathfrak{X}_{n}}\right)=\infty$ for the metric space $\left(\mathfrak{X}_{n}, d_{\mathfrak{X}_{n}}\right)$.

The suspension construction for pseudogroups given in 98 constructs a 2-dimensional matchbox manifold $\mathfrak{M}_{n}$ whose holonomy pseudogroup is $\mathcal{G}_{n}$. Thus, combining Theorem 8.2 with Corollary 4.5 and Propositions 3.4 and 3.5, we have the consequence:

THEOREM 8.3. For $n \geq 2$, the transitive Lipschitz matchbox manifold $\mathfrak{M}_{n}$ is not homeomorphic to an invariant subset of any $C^{1}$-foliation $\mathcal{F}_{M}$ of a manifold $M$.

Now consider a minimal Cantor action $\varphi_{2}: \mathfrak{X}_{2} \rightarrow \mathfrak{X}_{2}$ for some Cantor set $\mathfrak{X}_{2}$. For example, let $\varphi_{2}$ be a Denjoy type homeomorphism. Then there is a homeomorphism $h: \mathfrak{X}_{n} \rightarrow \mathfrak{X}_{2}$ and we can form the fusion of the action of $\mathcal{G}_{\mathfrak{X}_{n}}$ with that of $\varphi_{2}$. That is, we adjoin the action of $\widehat{\varphi}_{2} \equiv h^{-1} \circ \widehat{\varphi}_{2} \circ h$ to the action of $\mathcal{G}_{\mathfrak{X}_{n}}$ on $\mathfrak{X}_{n}$ to obtain a minimal action of the fusion pseudogroup, denoted by $\widehat{\mathcal{G}}_{\mathfrak{X}_{n}}$. Let $\widehat{\mathfrak{M}}_{n}$ denote the suspension matchbox manifold obtained from $\widehat{\mathcal{G}}_{\mathfrak{X}_{n}}$.

The action of $\widehat{\varphi}_{2}$ is not assumed to be Lipschitz, but we have in any case:

THEOREM 8.4. For $n \geq 2$, the minimal matchbox manifold $\widehat{\mathfrak{M}}_{n}$ is not homeomorphic to an invariant subset of any $C^{1}$-foliation $\mathcal{F}_{M}$ of a manifold $M$.

Proof. Suppose that $\widehat{\mathfrak{M}}_{n}$ is homeomorphic to an invariant subset $\mathcal{Z} \subset M$ of a $C^{1}$-foliation $\mathcal{F}_{M}$ on $M$. Then $\mathcal{Z}$ must be a saturated subset, and every leaf is dense as this is true for $\widehat{\mathfrak{M}}_{n}$. Moreover, the transversals to $\widehat{\mathfrak{M}}_{n}$ are Cantor sets, so $\mathcal{Z}$ must be an exceptional minimal set for $\mathcal{F}_{M}$. Then by Proposition 4.2 the embedding induces a metric $d_{\mathfrak{X}_{n}}^{\prime}$ on $\mathfrak{X}_{n}$ such that $\widehat{\mathcal{G}}_{\mathfrak{X}_{n}}$ is a Lipschitz pseudogroup for this metric. By construction, $\widehat{\mathcal{G}}_{\mathfrak{X}_{n}}$ contains $\mathcal{G}_{\mathfrak{X}_{n}}$ as a sub-pseudogroup, and so

$$
h\left(\widehat{\mathcal{G}}_{\mathfrak{X}_{n}}, d_{\mathfrak{X}_{n}}^{\prime}\right) \geq h\left(\mathcal{G}_{\mathfrak{X}_{n}}, d_{\mathfrak{X}_{n}}^{\prime}\right)=h\left(\mathcal{G}_{\mathfrak{X}_{n}}, d_{\mathfrak{X}_{n}}\right)=\infty
$$

where we use Proposition 3.4, But this contradicts Corollary 4.5 .

These two examples suggests the following:

PROBLEM 8.5. Show that there is no metric $d_{\mathfrak{X}_{n}}^{\prime \prime}$ on $\mathfrak{X}_{n}$ for which the action of $\widehat{\mathcal{G}}_{\mathfrak{X}_{n}}$ is Lipschitz.

It seems very likely that this has a positive solution, that no such metric can exists, though the proof of this fact may require some new insights or techniques.

We conclude this section with another remark, and a question. Recall that Problem 1.4 asks for obstructions to the existence of an embedding $\iota: \mathfrak{M} \rightarrow M$ of a Lipschitz matchbox manifold as an exceptional minimal set for a $C^{1}$-foliation $\mathcal{F}$ on $M$. Such an embedding implies in particular that the transverse Cantor set $\mathfrak{X}$ admits a Lipschitz embedding into the Euclidean space $\mathbb{R}^{q}$. The question 
of when a metric space admits a Lipschitz embedding in $\mathbb{R}^{q}$ dates from the 1928 paper 22, and is certainly well-studied. For example, the doubling property in Definition 4.7 of Assouad [7, and the weakening of this condition by Olson and Robinson [117, prove embedding criteria for metrics. These are types of "asymptotic small-scale homogeneity" properties of the metric $d_{\mathfrak{X}}$, which suggests an alternate approach to the Lipschitz embedding problem for minimal pseudogroups.

PROBLEM 8.6. Let $\mathfrak{X}$ be a Cantor space with metric $d_{\mathfrak{X}}$. Let $\mathcal{G}_{\mathfrak{X}}$ be a compactly-generated pseudogroup acting minimally on $\mathfrak{X}$, and which is Lipschitz with respect to $d_{\mathfrak{X}}$. If the metric $d_{\mathfrak{X}}$ satisfies some version of the doubling condition, so that $\left(\mathfrak{X}, d_{\mathfrak{X}}\right)$ admits a Lipschitz embedding into some $\mathbb{R}^{q}$, does there also exists an embedding such that $\mathcal{G}_{\mathfrak{X}}$ is obtained by the restriction of some $C^{1}$-pseudogroup acting on an open neighborhood of the embedded Cantor set?

For a Cantor set $\mathfrak{X}$ with an ultrametric $d_{\mathfrak{X}}$, the Lipschitz embedding problem for $\left(\mathfrak{X}, d_{\mathfrak{X}}\right)$ has been solved for various special cases. The work of Julien and Savinien in [84] estimates the Hausdorff dimension for a self-similar Cantor set with an ultrametric, and they derive estimates for its Lipschitz embedding dimension. The embedding properties of ultrametrics on Cantor sets which are the boundary of a hyperbolic group are discussed by Buyalo and Schroeder in [24, Chapter 8]. In both of these cases, it seems likely that the answer to Problem 8.6 is positive. In general, one expects the solution to be more complicated, as is almost always the case with Cantor sets.

Finally, recall that every Cantor set embeds homeomorphically to a Cantor set in $\mathbb{R}^{2}$, and any two such are homeomorphic by a homeomorphism of $\mathbb{R}^{2}$ restricted to the set. This classical fact, due to Brouwer, is proved in detail by Moise in Chapter 12 of [113. It has been used to construct topological embeddings of solenoids in codimension-two foliations, as in the work of Clark and Fokkink 34.

On the other hand, the tameness property of Cantor sets in $\mathbb{R}^{2}$ does not hold for all Cantor sets embedded in $\mathbb{R}^{3}$. The Antoine's Necklace is the classical example of this, as discussed in Chapter 18 of [113], and in Section 4.6 of [69. It seems natural to ask the naive question:

PROBLEM 8.7. Let $\mathfrak{A}$ denote the Antoine Cantor set embedded in $\mathbb{R}^{3}$, with the metric $d_{\mathfrak{A}}$ on $\mathfrak{A}$ induced by the restriction of the Euclidean metric. Does there is some exceptional minimal set for a $C^{1}$-foliation of codimension three, whose transverse model space is Lipschitz equivalent to $\left(\mathfrak{A}, d_{\mathfrak{A}}\right)$ ?

\section{Classification of Lipschitz solenoids}

In this section, we define Morita equivalence and Lipschitz equivalence of minimal pseudogroups, and consider the problem of Lipschitz classification for the special case of normal solenoids. While the condition of Morita equivalence is well-known and studied, Lipschitz equivalence seems less commonly studied, except possibly for group and semi-group actions on their boundaries.

Let $\mathcal{G}_{\mathfrak{X}}$ be a minimal pseudogroup acting on a Cantor space $\mathfrak{X}$, and let $V \subset \mathfrak{X}$ be a clopen subset. The induced pseudogroup $\mathcal{G}_{\mathfrak{X}} \mid V$ is defined as the subcollection of all maps in $\mathcal{G}_{\mathfrak{X}}$ with domain and range in $V$. The following is then the adaptation of the notion of Morita equivalence of groupoids, as in Haefliger [62, to the context of minimal Cantor actions.

DEFINITION 9.1. Let $\mathcal{G}_{\mathfrak{X}}$ be a minimal pseudogroup action on the Cantor set $\mathfrak{X}$ via Lipschitz homeomorphisms with respect to the metric $d_{\mathfrak{X}}$. Likewise, let $\mathcal{G}_{\mathfrak{Y}}$ be a minimal pseudogroup action on the Cantor set $\mathfrak{Y}$ via Lipschitz homeomorphisms with respect to the metric $d_{\mathfrak{Y}}$. Then

(1) $\left(\mathcal{G}_{\mathfrak{X}}, \mathfrak{X}, d_{\mathfrak{X}}\right)$ is Morita equivalent to $\left(\mathcal{G}_{\mathfrak{Y}}, \mathfrak{Y}, d_{\mathfrak{Y}}\right)$ if there exist clopen subsets $V \subset \mathfrak{X}$ and $W \subset \mathfrak{Y}$, and a homeomorphism $h: V \rightarrow W$ which conjugates $\mathcal{G}_{\mathfrak{X}} \mid V$ to $\mathcal{G}_{\mathfrak{Y}} \mid W$.

(2) $\left(\mathcal{G}_{\mathfrak{X}}, \mathfrak{X}, d_{\mathfrak{X}}\right)$ is Lipschitz equivalent to $\left(\mathcal{G}_{\mathfrak{Y}}, \mathfrak{Y}, d_{\mathfrak{Y}}\right)$ if the conjugation $h$ is Lipschitz.

Morita equivalence is sometimes called return equivalence in the literature [3, 53, 41].

Morita equivalence is a basic notion for the study of $C^{*}$-algebra invariants for foliation groupoids, as discussed by Renault [124] and Connes [43. Lipschitz equivalence is a basic notion for the study of metric non-commutative geometry [43]. 
The strongest results for classification, up to Morita equivalence, have been obtained for 1-dimensional minimal matchbox manifolds. Fokkink showed in his thesis [53] (see also Barge and Williams [10]) that if $f_{1}, f_{2}$ are $C^{1}$-actions on $\mathbb{S}^{1}$, each of which have Cantor minimal sets, then the induced minimal Cantor actions are Morita equivalent if and only if they have rotation numbers which are conjugate under the linear fractional action of $S L(2, \mathbb{Z})$ on $\mathbb{R}$. This implies there are uncountably many non-homeomorphic minimal matchbox manifolds which embed as minimal sets for $C^{1}$-foliations of $\mathbb{T}^{2}$. There is a higher-dimensional version of this result for torus-like matchbox manifolds, proved in [41. See the papers [11, 12, 13] for the classification of 1-dimensional minimal matchbox manifolds embedded in compact surfaces, which are necessarily not solenoids.

In general, the classification problem modulo orbit equivalence is unsolvable for the pseudogroups associated to minimal matchbox manifolds of dimension $n \geq 2$, as already for the normal solenoids with base manifold $\mathbb{T}^{n}$ where $n \geq 2$, they are not classifiable. See [70, 86, 140, 141] for discussions of the undecidability of the Borel classification problem up to orbit equivalence.

The advantage of considering Lipschitz equivalence of pseudogroup actions, is that while the equivalence is more refined, it can also be more practical to determine when two actions are not Lipschitz equivalent. We discuss the difference between Morita and Lipschitz classification in the case of the weak solenoids, where there are a well-known criteria for Morita equivalence.

First, we recall the criteria for when two weak solenoids are homeomorphic, as described in 38, Section 9], based on a result of using a result of Mioduszewski [107. Assume that we are given two presentations, where all spaces $\left\{M_{\ell} \mid \ell \geq 0\right\}$ and $\left\{N_{\ell} \mid \ell \geq 0\right\}$ are compact oriented manifolds, and all bonding maps are orientation-preserving coverings,

$$
\mathcal{P}=\left\{p_{\ell+1}: M_{\ell+1} \rightarrow M_{\ell} \mid \ell \geq 0\right\} \quad, \quad \mathcal{Q}=\left\{q_{\ell+1}: N_{\ell+1} \rightarrow N_{\ell} \mid \ell \geq 0\right\}
$$

which define weak solenoids $\mathcal{S}_{\mathcal{P}}$ and $\mathcal{S}_{\mathcal{Q}}$ as in (15), respectively. Choose basepoints $\bar{x} \in \mathcal{S}_{\mathcal{P}}$ and $\bar{y} \in \mathcal{S}_{\mathcal{Q}}$. We consider the special case where $M_{0}=N_{0}$, as the more general case easily reduces to this one, and the key issues are more evident in this special case. Let $\Pi_{\ell}^{\mathcal{P}}: \mathcal{S}_{\mathcal{P}} \rightarrow M_{\ell}$ denote the fibration map onto the factor $M_{\ell}$ for $\mathcal{S}_{\mathcal{P}}$, and $\Pi_{\ell}^{\mathcal{Q}}: \mathcal{S}_{\mathcal{Q}} \rightarrow N_{\ell}$ that for $\mathcal{S}_{\mathcal{Q}}$.

We can assume that $x_{0}=y_{0}$ in $M_{0}$, where $x_{0}=\Pi_{0}^{\mathcal{P}}(\bar{x})$ and $y_{0}=\Pi_{0}^{\mathcal{Q}}(\bar{y})$, then set $\mathcal{H}_{0}=\pi_{1}\left(M_{0}, x_{0}\right)$, where we suppress the dependence on basepoints. Define the subgroups $\mathcal{H}_{\ell} \subset \mathcal{H}_{0}$ which are the images of the groups $\pi_{1}\left(M_{\ell}, x_{\ell}\right)$ under the maps $\left(q_{\ell}\right)_{\#}$ associated to $\mathcal{P}$, and let $\mathcal{G}_{\ell} \subset \mathcal{H}_{0}$ be the corresponding images of the groups $\pi_{1}\left(N_{\ell}, y_{\ell}\right)$. Then we obtain two nested sequences of subgroups

$$
\begin{array}{cccccccccc}
\subset & \mathcal{H}_{\ell+1} & \subset & \mathcal{H}_{\ell} & \subset & \cdots & \subset & \mathcal{H}_{1} & \subset & \mathcal{H}_{0} \\
\subset & \mathcal{G}_{\ell+1} & \subset & \mathcal{G}_{\ell} & \subset & \cdots & \subset & \mathcal{G}_{1} & \subset & \mathcal{G}_{0}
\end{array}
$$

The proof of the following result can be found in the papers [105, 107, 125, 131.

THEOREM 9.2. The weak solenoids $\mathcal{S}_{\mathcal{P}}$ and $\mathcal{S}_{\mathcal{Q}}$ are basepoint homeomorphic if and only if there exists $\ell_{0} \geq 0$ and $\nu_{0} \geq 0$, such that for every $\ell \geq \ell_{0}$ there exists $\nu_{\ell} \geq \nu_{0}$ with $\mathcal{G}_{\nu_{\ell}} \subset \mathcal{H}_{\ell}$, and for every $\nu \geq \nu_{0}$ there exists $\ell_{\nu} \geq \ell_{0}$ with $\mathcal{H}_{\ell_{\nu}} \subset \mathcal{G}_{\nu}$.

The condition on bonding maps in Theorem 9.2 is called tower equivalence of the subgroup chains.

Let $\mathfrak{X}$ denote the fiber of $\Pi_{0}^{\mathcal{P}}$ over $\bar{x}$, and $\mathfrak{Y}$ the fiber of $\Pi_{0}^{\mathcal{Q}}$ over $\bar{y}$. Then the monodromy of the fibration $\Pi_{0}^{\mathcal{P}}$ defines the actions of $\mathcal{H}_{0}$ on $\mathfrak{X}$, and the action of $\mathcal{H}_{0}=\mathcal{G}_{0}$ on $\mathfrak{Y}$ is defined by the monodromy of $\Pi_{0}^{\mathcal{Q}}$. Then results of Clark, Lukina and the author yield:

THEOREM 9.3 ([38]). If the weak solenoids $\mathcal{S}_{\mathcal{P}}$ and $\mathcal{S}_{\mathcal{Q}}$ are basepoint homeomorphic, with $M_{0}=$ $N_{0}$, then the holonomy actions of $\mathcal{H}_{0}$ on $\mathfrak{X}$ and on $\mathfrak{Y}$ are Morita equivalent.

THEOREM 9.4 (41]). If the weak solenoids $\mathcal{S}_{\mathcal{P}}$ and $\mathcal{S}_{\mathcal{Q}}$ have base manifold $M_{0}=N_{0}=\mathbb{T}^{n}$, and the holonomy actions of $\mathcal{H}_{0}$ on $\mathfrak{X}$ and on $\mathfrak{Y}$ are Morita equivalent, then $\mathcal{S}_{\mathcal{P}}$ and $\mathcal{S}_{\mathcal{Q}}$ are basepoint homeomorphic. 
It follows that the classification problem for matchbox manifolds which are homeomorphic to a normal solenoid with base $\mathbb{T}^{n}$, reduces to the study of the Morita equivalence class of their holonomy pseudogroups, which by Theorem 9.2 reduces to a problem concerning the tower equivalence of subgroup chains in $\mathbb{Z}^{n}$. The classification problem for subgroup chains is not Borel, for $n \geq 2$.

In the case of classical Vietoris solenoids, where $M_{0}=\mathbb{S}^{1}$ and $\mathcal{H}_{0}=\mathbb{Z}$, the classification is much more straightforward. For each $\ell>0$ there exists integers $m_{\ell}>1$ and $n_{\ell}>1$, defined recursively, so that $\mathcal{H}_{\ell}=\left\langle m_{1} m_{2} \cdots m_{\ell}\right\rangle \subset \mathbb{Z}$, and $\mathcal{G}_{\ell}=\left\langle n_{1} n_{2} \cdots n_{\ell}\right\rangle \subset \mathbb{Z}$. Let $P$ be the set of all prime factors of the integers $\left\{m_{\ell} \mid \ell>0\right\}$, included with multiplicity, and let $Q$ be the same for the integers $\left\{n_{\ell} \mid \ell>0\right\}$. For example, for the dyadic solenoid, the set $P=\{2,2,2, \ldots\}$ is an infinite collection of copies of the prime 2. These infinite sets of primes $P$ and $Q$ are ordered by the sequence in which they appear in the factorizations of the covering degrees $m_{\ell}$ and $n_{\ell}$.

If the two sets $P$ and $Q$ are in bijective correspondence, then it is an exercise to show that the tower equivalence condition of Theorem 9.2 is satisfied for the presentations $\mathcal{P}$ and $\mathcal{Q}$, which yields the classification of Vietoris solenoids up to homeomorphism by Bing [16] and McCord [105] (see also Aarts and Fokkink [1]), and also the classification up to Morita equivalence of the associated minimal $\mathbb{Z}$-actions on the Cantor set fibers.

However, for the metrics on the Cantor sections $\mathfrak{X} \subset \mathfrak{M}=\mathcal{S}_{\mathcal{P}}$ and $\mathfrak{Y} \subset \mathfrak{N}=\mathcal{S}_{\mathcal{P}}$ as defined by the formula in (20), it is evident that if the bijection $\sigma: P \leftrightarrow Q$ permutes the elements by increasingly large degrees with respect to their ordering, then the induced map between the fibers, $h_{\sigma}: \mathfrak{X} \cong \mathfrak{Y}$, will not be Lipschitz. This motivates introducing the following invariant of a tower of equivalences.

Let $\mathcal{P}$ and $\mathcal{Q}$ be presentations with common base manifold $M_{0}$, and suppose there exists a tower equivalence between them. That is, there exists $\ell_{0} \geq 0$ and $\nu_{0} \geq 0$, such that for every $\ell \geq \ell_{0}$ there exists $\nu_{\ell} \geq \nu_{0}$ with $\mathcal{G}_{\nu_{\ell}} \subset \mathcal{H}_{\ell}$, and for every $\nu \geq \nu_{0}$ there exists $\ell_{\nu} \geq \ell_{0}$ with $\mathcal{H}_{\ell_{\nu}} \subset \mathcal{G}_{\nu}$. Define the displacement of these indexing functions $\ell \mapsto \nu_{\ell}$ and $\nu \mapsto \ell_{\nu}$ to be

$$
\operatorname{Disp}\left(\ell_{\nu}, \nu_{\ell}\right)=\max \left\{\sup \left\{\left|\ell_{\nu}-\nu\right| \mid \nu \geq \nu_{0}\right\}, \sup \left\{\left|\nu_{\ell}-\ell\right| \mid \ell \geq \ell_{0}\right\}\right\}
$$

If $\operatorname{Disp}\left(\ell_{\nu}, \nu_{\ell}\right)<\infty$, then we say that $\mathcal{P}$ and $\mathcal{Q}$ are bounded tower equivalent.

THEOREM 9.5. Let $\mathcal{P}$ and $\mathcal{Q}$ be presentations with common base manifold $M_{0}$, and suppose there exists a tower equivalence between them, defined by maps $\ell \mapsto \nu_{\ell}$ and $\nu \mapsto \ell_{\nu}$. Let the fiber metrics be defined by the formula (201) with $a_{\ell}=3^{-\ell}$. Then the action of $\mathcal{H}_{0}$ on the fiber $\mathfrak{X}$ of $\Pi_{0}^{\mathcal{P}}$ is Lipschitz equivalent to the action of $\mathcal{H}_{0}$ on the fiber $\mathfrak{Y}$ of $\Pi_{0}^{\mathcal{Q}}$ if and only if $\mathcal{P}$ and $\mathcal{Q}$ are bounded tower equivalent.

The proof that $\operatorname{Disp}\left(\ell_{\nu}, \nu_{\ell}\right)<\infty$ implies Lipschitz equivalence for the metrics defined by (20) with $a_{\ell}=3^{-\ell}$ is an exercise in the definitions, using the expression (17) for the metric on the fibers. The converse direction, that Lipschitz equivalence implies bounded tower equivalence, follows from the works of Miyata and Watanabe [108, 109.

We give a simple example of Theorem 9.5 in the case of Vietoris solenoids. With the notation as above, suppose the the covering degrees $m_{\ell}$ for the presentation $\mathcal{P}$ with base $M_{0}=\mathbb{S}^{1}$ are given by $m_{\ell}=2$ for $\ell$ odd, and $m_{\ell}=3$ for $\ell$ even. Let the covering degrees for the presentation $\mathcal{Q}$ be given by the sequence $\left\{n_{1}, n_{2}, n_{3}, \ldots\right\}=\{2,3,2,2,3,2,2,2,2,3, \ldots\}$. In general, the $\ell$-th cover of degree 3 is followed by $2^{\ell}$ covers of degree 2 . Then these two sequences are clearly tower equivalent, but their displacement is infinite. It follows that the matchbox manifolds $\mathfrak{M}=\mathcal{S}_{\mathcal{P}}$ and $\mathfrak{N}=\mathcal{S}_{\mathcal{P}}$ are homeomorphic, but are not Lipschitz equivalent.

\section{REFERENCES}

[1] J.M. Aarts and R. Fokkink, The classification of solenoids, Proc. Amer. Math. Soc., 111:1161-1163, 1991.

[2] J. Aarts and L. Oversteegen, Flowbox manifolds, Trans. Amer. Math. Soc., 327:449-463, 1991.

[3] J. Aarts and L. Oversteegen, Matchbox manifolds, In Continua (Cincinnati, OH, 1994), Lecture Notes in Pure and Appl. Math., Vol. 170, Dekker, New York, 1995, pages 3-14..

[4] J.M. Aarts and M. Martens, Flows on one-dimensional spaces, Fund. Math., 131:39-58, 1988.

[5] J. Alvarez Lopez and M. Moreira Galicia, Topological Molino's theory, preprint, 2013; arXiv:1307.1276 
[6] J. Anderson and I. Putnam, Topological invariants for substitution tilings and their associated $C^{*}$-algebras, Ergodic Theory Dyn. Syst., 18:509-537, 1998.

[7] P. Assouad, Plongements lipschitziens dans $\mathbf{R}^{n}$, Bull. Soc. Math. France, 111:429-448, 1983.

[8] O. Attie and S. Hurder, Manifolds which cannot be leaves of foliations, Topology, 35(2):335-353, 1996.

[9] R. Baer, Abelian groups without elements of finite order, Duke Math. Jour., 3:68-122, 1937.

[10] M. Barge and R. Williams, Classification of Denjoy continua, Topology Appl., 106:77-89, 2000.

[11] M. Barge and B. Diamond, A complete invariant for the topology of one-dimensional substitution tiling spaces, Ergodic Theory Dynam. Systems, 21:1333-1358, 2001.

[12] M. Barge and R.C. Swanson, New techniques for classifying Williams solenoids, Tokyo J. Math., 30:139-157, 2007.

[13] M. Barge and B. Martensen, Classification of expansive attractors on surfaces, Ergodic Theory Dynam. Systems, 31:1619-1639, 2011.

[14] J. Bellissard and A. Julien, Bi-Lipshitz Embedding of Ultrametric Cantor Sets into Euclidean Spaces, preprint, February, 2012, arXiv:1202.4330.

[15] R. Benedetti and J.-M. Gambaudo, On the dynamics of $\mathbb{G}-$ solenoids. Applications to Delone sets, Ergodic Theory Dyn. Syst., 23:673-691, 2003.

[16] R.H. Bing, A simple closed curve is the only homogeneous bounded plane continuum that contains an arc, Canad. J. Math., 12:209-230, 1960.

[17] A. Biś, S. Hurder, and J. Shive, Hirsch foliations in codimension greater than one, In Foliations 2005, World Scientific Publishing Co. Inc., River Edge, N.J., 2006: 71-108.

[18] A. Biś and M. Urbanski, Geometry of Markov systems and codimension one foliations, Ann. Polon. Math., 94:187-196, 2008.

[19] E. Blanc, Laminations minimales résiduellement à 2 bouts, Comment. Math. Helv., 78:845-864, 2003.

[20] M. Bonk and O. Schramm, Embeddings of Gromov hyperbolic spaces, Geom. Funct. Anal., 10:266-306, 2000.

[21] K. Borsuk, Concerning homotopy properties of compacta, Fund. Math., 62:223-254, 1968.

[22] M.G. Bouligand, Ensembles impropres et nombre dimensionnel, Bull. Sci. Math., 1928.

[23] R. Bowen, Entropy for group endomorphisms and homogeneous spaces, Trans. Amer. Math. Soc., 153:401414, 1971.

[24] S. Buyalo and V. Schroeder, Elements of asymptotic geometry, EMS Monographs in Mathematics, European Mathematical Society (EMS), Zürich, 2007.

[25] C. Camacho and A. Lins Neto, Geometric Theory of Foliations, Translated from the Portuguese by Sue E. Goodman, Progress in Mathematics, Birkhäuser Boston, MA, 1985.

[26] A. Candel and L. Conlon, Foliations I, Amer. Math. Soc., Providence, RI, 2000.

[27] J. Cantwell and L. Conlon, Leaves with isolated ends in foliated 3-manifolds, Topology, 16:311-322, 1977.

[28] J. Cantwell and L. Conlon, Growth of leaves, Comment. Math. Helv., 53:93-111, 1978.

[29] J. Cantwell and L. Conlon, Every surface is a leaf, Topology, 26:265-285, 1987.

[30] J. Cantwell and L. Conlon, Foliations and subshifts, Tohoku Math. J. (2), 40:165-187, 1988.

[31] J. Cantwell and L. Conlon, Endsets of exceptional leaves; a theorem of G. Duminy, In Foliations: Geometry and Dynamics (Warsaw, 2000), World Scientific Publishing Co. Inc., River Edge, N.J., 2002:225-261.

[32] J. Cantwell and L. Conlon, An interesting class of $C^{1}$ foliations, Topology Appl., 126:281-297, 2002.

[33] D. Cass, Minimal leaves in foliations, Trans. Amer. Math. Soc., 287:201-213, 1985.

[34] A. Clark and R. Fokkink, Embedding solenoids, Fund. Math., 181:111-124, 2004.

[35] A. Clark and M. Sullivan, The linking homomorphism of one-dimensional minimal sets, Topology Appl., 141:125-145, 2004.

[36] A. Clark, R. Fokkink and O. Lukina, The Schreier continuum and ends, Houston J. Math. 40(2):569-599, 2014; arXiv:1007.0746v1.

[37] A. Clark and S. Hurder, Embedding solenoids in foliations, Topology Appl., 158:1249-1270, 2011.

[38] A. Clark and S. Hurder, Homogeneous matchbox manifolds, Trans. Amer. Math. Soc., 365:3151-3191, 2013; arXiv:1006.5482v2.

[39] A. Clark, S. Hurder and O. Lukina, Voronoi tessellations for matchbox manifolds, Topology Proceedings, 41:167-259, 2013; arXiv:1107.1910v2.

[40] A. Clark, S. Hurder and O. Lukina, Shape of matchbox manifolds, Indagationes Mathematicae, 25(4):669-712, 2014; arXiv:1308.3535.

[41] A. Clark, S. Hurder and O. Lukina, Classifying matchbox manifolds, submitted, November 2013; arXiv:1311.0226.

[42] A. Clark, S. Hurder and O. Lukina, Y-like matchbox manifolds, in preparation, 2014.

[43] A. Connes, Noncommutative Geometry, Academic Press, San Diego, CA, 1994.

[44] D. Cooper and T. Pignataro, On the shape of Cantor sets, J. Differential Geom., 28:203-221, 1988.

[45] J.-M. Cordier and T. Porter, Shape theory: Categorical methods of approximation, Ellis Horwood Ltd., Chichester, 1989.

[46] S. Crovisier and M. Rams, IFS attractors and Cantor sets, Topology Appl., 153:1849-1859, 2006.

[47] D. D'Angeli, A. Donno, M. Matter, and T. Nagnibeda, Schreier graphs of the Basilica group, J. Mod. Dyn., 4:167-205, 2010.

[48] A. Denjoy, Sur les courbes définies par des équations différentielles à la surface du tore, J. Math. Pures et Appl., 11:333-375, 1932. 
[49] B. Deroin, V. Kleptsyn and A. Navas, Sur la dynamique unidimensionnelle en régularité intermédiaire, Acta Math., 199:199-262, 2007.

[50] M. do Carmo, Riemannian geometry, Translated from the second Portuguese edition by Francis Flaherty, Birkhäuser Boston Inc., Boston, 1992.

[51] G. Duminy and V. Sergiescu, Sur la nullité de Godbillon-Vey, C. R. Acad. Sci. Paris Sér. I Math., 292:821824, 1981.

[52] G. A. Edgar, Measure, Topology and Fractal Geometry, Springer-Verlag, Undergraduate Texts in Mathematics, Harrissonburg, Virginia, 1990.

[53] R. Fokkink, The structure of trajectories, Ph. D. Thesis, TU Delft, 1991.

[54] F. Fokkink and L. Oversteegen, Homogeneous weak solenoids, Trans. Amer. Math. Soc., 354:3743-3755, 2002.

[55] J.-M. Gambaudo, D. Sullivan and C. Tresser, Infinite cascades of braids and smooth dynamical systems, Topology, 33:85-94, 1994.

[56] F. Gardner and D. Sullivan, Symmetric structures on a closed curve, Amer. J. Math., 114:683-736, 1992.

[57] K. Gelfert and M. Rams, Geometry of limit sets for expansive Markov systems, Trans. Amer. Math. Soc., 361:2001-2020, 2009.

[58] É. Ghys, Une variété qui n'est pas une feuille, Topology, 24:67-73, 1985.

[59] É. Ghys, R. Langevin, and P. Walczak, Entropie geometrique des feuilletages, Acta Math., 168:105-142, 1988.

[60] É Ghys, Laminations par surfaces de Riemann, in Dynamique et Géométrie Complexes, Panoramas \& Synthèses, 8:49-95, 1999.

[61] A. Haefliger, Structures feulletées et cohomologie à valeur dans un faisceau de groupoïdes, Comment. Math. Helv., 32:248-329, 1958.

[62] A. Haefliger, Groupö̈des d'holonomie et classifiants, In Transversal structure of foliations (Toulouse, 1982), Asterisque, 177-178, Société Mathématique de France, 1984:70-97.

[63] J. Harrison, $C^{2}$-counterexamples to the Seifert conjecture, Topology, 27:249-278, 1988.

[64] J. Harrison, Denjoy fractals, Topology, 28:59-80, 1989.

[65] G. Hector and U. Hirsch, Introduction to the Geometry of Foliations, Parts A,B, Vieweg, Braunschweig, 1981.

[66] G. Hector, Architecture des feuilletages de classe $C^{2}$, Third Schnepfenried geometry conference, Vol. 1 (Schnepfenried, 1982), Astérisque, 107, Société Mathématique de France 1983, 243-258.

[67] S. Helgason, Differential geometry, Lie groups, and symmetric spaces, Pure and Applied Mathematics, Vol. 80, Academic Press Inc., New York, 1978.

[68] M. Hirsch, A stable analytic foliation with only exceptional minimal sets, in Dynamical Systems, Warwick, 1974, Lect. Notes in Math. vol. 468, , Springer-Verlag, 1975, 9-10.

[69] J.G. Hocking and G.S. Young, Topology Dover Publications, Inc., New York, 1988.

[70] G. Hjorth, Classification and orbit equivalence relations, Mathematical Surveys and Monographs, Vol. 75, Amer. Math. Soc., Providence, RI, 2000.

[71] J. Hu and D. Sullivan, Topological conjugacy of circle diffeomorphisms, Ergodic Theory Dynam. Systems, 17:173-186, 1997.

[72] B. Hughes, Trees and ultrametric spaces: a categorical equivalence, Adv. Math., 189:148-191, 2004.

[73] B. Hughes, Á. Martínez-Pérez and M. Morón, Bounded distortion homeomorphisms on ultrametric spaces, Ann. Acad. Sci. Fenn. Math., 35:473-492, 2010.

[74] B. Hughes, Trees, ultrametrics, and noncommutative geometry, Pure Appl. Math. Q., 8:221-312, 2012.

[75] S. Hurder and A. Katok, Differentiability, rigidity and Godbillon-Vey classes for Anosov flows, Publ. Math. Inst. Hautes Etudes Sci., 72:5-61, 1991.

[76] S. Hurder, Dynamics and the Godbillon-Vey class: a History and Survey, In Foliations: Geometry and Dynamics (Warsaw, 2000), World Scientific Publishing Co. Inc., River Edge, N.J., 2002:29-60.

[77] S. Hurder, Classifying foliations, Foliations, Geometry and Topology. Paul Schweitzer Festschrift, (eds. Nicoalu Saldanha et al), Contemp. Math. Vol. 498, American Math. Soc., Providence, RI, 2009, pages 1-61.

[78] S. Hurder, Lectures on Foliation Dynamics: Barcelona 2010, Foliations: Dynamics, Geometry and Topology, Advanced Courses in Mathematics CRM Barcelona, to appear 2014.

[79] S. Hurder and A. Rechtman, Dynamics of generic Kuperberg flows, to appear, 2014; arXiv:1306.5025

[80] S. Hurder and O. Lukina, Coarse entropy and transverse dimension, in preparation, 2014.

[81] S. Hurder and C. Meniño Cotón, LS category of foliations and Fölner properties, in preparation, 2014.

[82] T. Inaba, T. Nishimori, M. Takamura and N. Tsuchiya, Open manifolds which are nonrealizable as leaves, Kodai Math. J., 8:112-119, 1985

[83] T. Januszkiewicz, Characteristic invariants of noncompact Riemannian manifolds, Topology 23:289-301, 1984.

[84] A. Julien and J. Savinien, Embeddings of self-similar ultrametric Cantor sets, Topology Appl. 16:2148-2157, 2011.

[85] I. Kapovich and N. Benakli, Boundaries of hyperbolic groups, In Combinatorial and geometric group theory (New York, 2000/Hoboken, NJ, 2001), Contemp. Math., Vol. 296, pages 39-93, Amer. Math. Soc., Providence, RI, 2000.

[86] A. Kechris and B. Miller, Topics in orbit equivalence, Lect. Notes in Math., Vol. 1852, Springer, Berlin, 2004.

[87] J. Kennedy and J. Yorke, Bizarre topology is natural in dynamical systems, Bull. Amer. Math. Soc. (N.S.), 32:309-316, 1995. 
[88] V. Kleptsyn and A. Navas, A Denjoy type theorem for commuting circle diffeomorphisms with derivatives having different Hölder differentiability classes, Mosc. Math. J., 8:477-492, 616, 2008.

[89] R.J. Knill, A $C^{\infty}$ flow on $S^{3}$ with a Denjoy minimal set, J. Differential Geom., 16:271-280, 1981.

[90] U. Lang and T. Schlichenmaier, Nagata dimension, quasisymmetric embeddings, and Lipschitz extensions, Int. Math. Res. Not., 58:3625-3655, 2005; arXiv:math/0410048.

[91] H.B. Lawson, Jr., Codimension-one foliations of spheres, Ann. of Math. (2), 94:494-503, 1971.

[92] H.B. Lawson, Jr., The Quantitative Theory of Foliations, NSF Regional Conf. Board Math. Sci., Vol. 27, 1977.

[93] F. Ledrappier and L.-S. Young, The metric entropy of diffeomorphisms. I. Characterization of measures satisfying Pesin's entropy formula, Ann. of Math. (2), 122:509-539, 1985.

[94] F. Ledrappier and L.-S. Young, The metric entropy of diffeomorphisms. II. Relations between entropy, exponents and dimension, Ann. of Math. (2), 122:540-574, 1985.

[95] Á. Lozano-Rojo, The Cayley foliated space of a graphed pseudogroup, in XIV Fall Workshop on Geometry and Physics, Publ. R. Soc. Mat. Esp., Vol. 10, pages 267-272, R. Soc. Mat. Esp., Madrid, 2006.

[96] Á. Lozano-Rojo, An example of a non-uniquely ergodic lamination, Ergodic Theory Dynam. Systems, 31:449-457, 2011.

[97] Á. Lozano-Rojo, Codimension zero laminations are inverse limits, Topology Appl., 160:341-349, 2013; arXiv:1204.6439.

[98] Á. Lozano-Rojo and O. Lukina, Suspensions of Bernoulli shifts, Dyn. Syst., 28:551-566, 2013; arXiv:1204.5376

[99] O. Lukina, Hierarchy of graph matchbox manifolds, Topology Appl., 159:3461-3485, 2012; arXiv:1107.5303v3.

[100] O. Lukina, Hausdorff dimension of graph matchbox manifolds, preprint, July 2014; arXiv:1407.0693.

[101] M. Lyubich and Y. Minsky, Laminations in holomorphic dynamics, J. Differential Geom., 47:17-94, 1997.

[102] J.M. Mackay and J. Tyson, Conformal dimension, University Lecture Series, Vol. 54, American Mathematical Society, Providence, RI, 2010.

[103] L. Markus and K. Meyer, Periodic orbits and solenoids in generic Hamiltonian dynamical systems, Amer. J. Math., 102:25-92, 1980.

[104] S. Matsumoto, Measure of exceptional minimal sets of codimension one foliations, In A Fête of Topology, Academic Press, Boston, 1988, 81-94.

[105] C. McCord, Inverse limit sequences with covering maps, Trans. Amer. Math. Soc., 114:197-209, 1965.

[106] D. McDuff, $C^{1}$-minimal subsets of the circle, Ann. Inst. Fourier (Grenoble), 31:177-193, 1981.

[107] J. Mioduszewski, Mappings of inverse limits, Colloq. Math., 10:39-44, 1963.

[108] T. Miyata and T. Watanabe, Lipschitz functions and approximate resolutions; Proceedings of the International Conference on Topology and its Applications (Yokohama, 1999), Topology Appl., 122:353-375, 2002.

[109] T. Miyata and T. Watanabe, Bi-Lipschitz maps and the category of approximate resolutions, Glas. Mat. Ser. III, 38(58):129-155, 2003.

[110] T. Miyata and T. Watanabe, Approximate resolutions and the fractal category, Glas. Mat. Ser. III, 38(58):377-393, 2003.

[111] T. Miyata and T. Watanabe, Approximate resolutions and box-counting dimension, Topology Appl., 132:49$69,2003$.

[112] T. Miyata, An approximate inverse system approach to shape fibrations, Glas. Mat. Ser. III, 44(64):215-240, 2009.

[113] E. Moise, Geometric topology in dimensions 2 and 3, Graduate Texts in Mathematics, Vol. 47, SpringerVerlag, New York, 1977.

[114] C.C. Moore and C. Schochet, Analysis on Foliated Spaces, With appendices by S. Hurder, Moore, Schochet and Robert J. Zimmer, Math. Sci. Res. Inst. Publ. vol. 9, Springer-Verlag, New York, 1988. Second Edition, Cambridge University Press, New York, 2006.

[115] C. Mouron, The solenoids are the only circle-like continua that admit expansive homeomorphisms, Fund. Math., 205:237-264, 2009.

[116] C. Mouron, The classification of circle-like continua that admit expansive homeomorphisms, Fund. Math., 211:101-133, 2011.

[117] E. Olson and J.C. Robinson, Almost bi-Lipschitz embeddings and almost homogeneous sets, Trans. Amer. Math. Soc., 362:145-168, 2010.

[118] P. Pansu, Dimension conforme et sphère à l'infini des variétés à courbure négative, Ann. Acad. Sci. Fenn. Ser. A I Math. 14:177-212, 1989.

[119] A. Phillips and D. Sullivan, Geometry of leaves, Topology 20:209-218, 1981.

[120] D. Pixton, Nonsmoothable, unstable group actions, Trans. Amer. Math. Soc., 229:259-268, 1977.

[121] N. Priebe Frank and L. Sadun, Topology of some tiling spaces without finite local complexity, Discrete Contin. Dyn. Syst., 23:847-865, 2009.

[122] H. Rao, H.-J. Ruan and L.-F. Xi, Lipschitz equivalence of self-similar sets, C. R. Math. Acad. Sci. Paris, 342:191-196, 2006.

[123] H. Rao, H.-J. Ruan and Y. Wang, Lipschitz equivalence of Cantor sets and algebraic properties of contraction ratios, Trans. Amer. Math. Soc., 364:1109-1126, 2012.

[124] J. Renault, A groupoid approach to $C^{*}$-algebras, Lecture Notes in Mathematics Vol. 793, Springer, Berlin, 1980. 
[125] J. Rogers, Jr., Inverse limits of manifolds with covering maps, In Topology Conference (Proc. General Topology Conf., Emory Univ., Atlanta, Ga., 1970), Dept. Math., Emory Univ., Atlanta, Ga., 1970, 81-85.

[126] D. Ruelle, Noncommutative algebras for hyperbolic diffeomorphisms, Invent. Math. 93:1-13, 1988.

[127] R. Sacksteder, Foliations and pseudogroups, Amer. J. Math., 87:79-102, 1965.

[128] L. Sadun and R.F. Williams, Tiling spaces are Cantor set fiber bundles, Ergodic Theory Dynam. Systems, 23:307-316, 2003.

[129] L. Sadun Tiling spaces are inverse limits, J. Math. Phys., 44:5410-5414, 2003.

[130] L. Sadun, Topology of tiling spaces, University Lecture Series, Vol. 46, American Mathematical Society, Providence, RI, 2008.

[131] R. Schori, Inverse limits and homogeneity, Trans. Amer. Math. Soc., 124:533-539, 1966.

[132] P.A. Schweitzer, Counterexamples to the Seifert conjecture and opening closed leaves of foliations, Ann. of Math. (2), 100:386-400, 1974.

[133] P.A. Schweitzer, Surfaces not quasi-isometric to leaves of foliations of compact 3-manifolds, in Analysis and geometry in foliated manifolds (Santiago de Compostela, 1994), World Sci. Publ., River Edge, NJ, 1995, pages 223-238.

[134] P.A. Schweitzer, Riemannian manifolds not quasi-isometric to leaves in codimension one foliations, Ann. Inst. Fourier (Grenoble), 61:1599-1631, 2011.

[135] F. Souza and P.A. Schweitzer, Manifolds that are not leaves of codimension one foliations, Internat. J. Math., 24: 2013; arXiv:1111.2766.

[136] S. Smale, Differentiable Dynamical Systems, Bull. Amer. Math. Soc., 73:747-817, 1967.

[137] J.D. Sondow, When is a manifold a leaf of some foliation?, Bull. Amer. Math. Soc., 81:622-624, 1975.

[138] D. Sullivan, Inside and outside manifolds, in Proceedings of the International Congress of Mathematicians (Vancouver, B. C., 1974), Vol. 1, Canad. Math. Congress, Montreal, Quebec, 1975, pages 201-207.

[139] D. Sullivan, Bounds, quadratic differentials, and renormalization conjectures, American Mathematical Society centennial publications, Vol. II (Providence, RI, 1988), pages 417-466, 1992.

[140] S. Thomas, On the complexity of the classification problem for torsion-free abelian groups of finite rank, Bull. Symbolic Logic, 7:329-344, 2001.

[141] S. Thomas, The classification problem for torsion-free abelian groups of finite rank, J. Amer. Math. Soc., 16:233-258, 2003.

[142] P. Tukia and J. Väisälä Quasisymmetric embeddings of metric spaces, Ann. Acad. Sci. Fenn. Ser. A I Math., 5:97-114, 1980.

[143] I. Tamura, Spinnable structures on differentiable manifolds, Proc. Japan Acad., 48:293-296, 1972.

[144] J. Tyson and J.-M. Wu Quasiconformal dimensions of self-similar fractals, Rev. Mat. Iberoam., 22:205-258, 2006.

[145] D. van Dantzig, Über topologisch homogene Kontinua, Fund. Math., 15:102-125, 1930.

[146] L. Vietoris, Über den höheren Zusammenhang kompakter Räume und eine Klasse von zusammenhangstreuen Abbildungen, Math. Ann., 97:454-472, 1927.

[147] P. Walczak, Dynamics of foliations, groups and pseudogroups, Instytut Matematyczny Polskiej Akademii Nauk. Monografie Matematyczne (New Series) [Mathematics Institute of the Polish Academy of Sciences. Mathematical Monographs (New Series)], Vol. 64. Birkhäuser Verlag, Basel, 2004.

[148] R.F. Williams, One-dimensional non-wandering sets, Topology, 6:473-487, 1967.

[149] R.F. Williams, Classification of one dimensional attractors, In Global Analysis (Proc. Sympos. Pure Math., Vol.XIV, Berkeley, Calif., 1968), American Mathematical Society, Providence, RI, 1970: 341-361.

[150] R.F. Williams, Expanding attractors, Inst. Hautes Études Sci. Publ. Math., 43:169-203, 1974.

[151] H. Winkelnkemper, Manifolds as open books, Bull. Amer. Math. Soc., 79:45-51, 1973.

[152] A. Zeghib, An example of a 2-dimensional no leaf, in Geometric study of foliations (Tokyo, 1993), World Sci. Publ., River Edge, NJ, 1994, pages 475-477.

[153] A. Zeghib, Lipschitz regularity in some geometric problems, Geom. Dedicata, 107:57-83, 2004.

Steven Hurder, Department of Mathematics, University of Illinois at Chicago, 322 SeO (m/c 249 ), 851 S. Morgan Street, Chicago, IL 60607-7045

E-mail address: hurder@uic.edu 